\title{
Ultrawideband Microwave Breast Cancer Detection: A Detection-Theoretic Approach Using the Generalized Likelihood Ratio Test
}

\author{
Shakti K. Davis*, Student Member, IEEE, Henri Tandradinata, Susan C. Hagness, Senior Member, IEEE, and \\ Barry D. Van Veen, Fellow, IEEE
}

\begin{abstract}
Microwave imaging has been suggested as a promising modality for early-stage breast cancer detection. In this paper, we propose a statistical microwave imaging technique wherein a set of generalized likelihood ratio tests (GLRT) is applied to microwave backscatter data to determine the presence and location of strong scatterers such as malignant tumors in the breast. The GLRT is formulated assuming that the backscatter data is Gaussian distributed with known covariance matrix. We describe the method for estimating this covariance matrix offline and formulating a GLRT for several heterogeneous two-dimensional (2-D) numerical breast phantoms, several three-dimensional (3-D) experimental breast phantoms, and a 3-D numerical breast phantom with a realistic half-ellipsoid shape. Using the GLRT with the estimated covariance matrix and a threshold chosen to constrain the false discovery rate (FDR) of the image, we show the capability to detect and localize small $(<0.6 \mathrm{~cm})$ tumors in our numerical and experimental breast phantoms even when the dielectric contrast of the malignant-to-normal tissue is below 2:1.
\end{abstract}

Index Terms-Array signal processing, biomedical electromagnetic imaging, breast cancer detection, clutter, FDTD methods, generalized likelihood ratio test (GLRT), microwave imaging, microwave measurements, tissue phantoms, ultrawide-band (UWB) radar.

\section{INTRODUCTION}

D ETECTING breast cancer in its earliest stages is looked upon as the best hope for successful treatment of the disease [1]. The limitations of conventional X-ray mammograms [1], [2] are well-recognized and in response to these limitations several complementary modalities for breast cancer are under investigation. Active microwave imaging is one promising alternative screening technology that is nonionizing, noninvasive, and does not require breast compression. For this modality, low-power electromagnetic waves that are transmitted into the

Manuscript received September 22, 2004; revised December 23, 2004. This work was supported by the National Institutes of Health under Grants R21 CA92188-01 and 1 F31 CA11094-01 both awarded by the National Cancer Institute, the Department of Defense Breast Cancer Research Program under Grant DAMD17-02-1-0625, the National Science Foundation under Grant BES 0201880, and the Graduate Engineering Research Scholars Program. Asterisk indicates corresponding author.

*S. K. Davis is with the Department of Electrical and Computer Engineering, University of Wisconsin, 1415 Engineering Drive, Madison, WI 53706 USA (email: skdavis@cae.wisc.edu).

H. Tandradinata is with ZS Associates, Evanston, IL 60201 USA

S. C. Hagness and B. D. Van Veen are with the Department of Electrical and Computer Engineering, University of Wisconsin, Madison, WI 53706 USA (e-mail: hagness@engr.wisc.edu; vanveen@engr.wisc.edu).

Digital Object Identifier 10.1109/TBME.2005.847528 breast undergo scattering or selective heating/absorption due to the dielectric contrast between malignant tumors and normal breast tissue at microwave frequencies [3]-[5]. The received signals are processed to extract information about the tissue dielectric-properties distribution or other tissue characteristics of the breast.

Current research in active microwave breast imaging can be divided into three categories: hybrid microwave-induced acoustic imaging [6]-[8], microwave tomography [9]-[16], and ultrawideband (UWB) radar techniques [17]-[27]. In the hybrid approach microwave signals are transmitted into the breast to heat tumors and ultrasound transducers detect the pressure waves generated by tumor expansion. In tomographic image reconstruction, a nonlinear inverse scattering problem is solved to recover an image of the dielectric properties in the breast. In contrast to the image recovery goal of tomography, the proposed UWB radar approach solves a simpler computational problem by seeking only to identify the presence and location of significant scatterers such as malignant breast tumors. In the UWB radar approach, high bandwidths and large antenna apertures are used to improve spatial resolution at microwave frequencies.

Previously UWB radar investigations have used beamforming techniques of varying complexity to synthetically focus scattered signals toward a point in the breast and calculate the corresponding scattered energy. Images obtained by beamforming are maps of the focused backscatter energy as a function of position where strong scatterers such as malignant tumors are identified as high-energy regions in the image. One method for creating a synthetic focal point is confocal imaging which employs simple delay-and-sum beamforming [17]-[24]. While confocal processing is computationally inexpensive, it does not account for dispersive propagation effects in breast tissue or fractional time delays and the simple filter design has limited ability to discriminate against artifacts and noise. An alternative focal technique, microwave imaging via space-time (MIST) beamforming [25]-[27], uses filters that compensate for dispersion and fractional time delays. The filters solve a penalized least-squares problem such that signals originating from a candidate tumor location are passed with approximately unit amplitude and linear phase while the white noise gain is constrained. This filter design dramatically improves clutter suppression and spatial discrimination compared to the earlier delay-and-sum beamformers. 
In this paper we approach the UWB radar problem of determining whether a tumor exists at a candidate location as a hypothesis testing problem. Hypothesis testing is widely used in radar applications to make optimal decisions concerning target presence or classification. We consider a statistical framework, formulating our tests as likelihood ratios (see for example [28]) which requires some assumptions about the distribution of the data. A simple Gaussian-distributed model is proposed to describe the data; however, we assume that the data model has some unknown parameters that must be estimated. The parameter estimates are substituted into the probability distributions for each hypothesis and the resulting generalized likelihood ratio test (GLRT) is used to detect a tumor at a candidate location in the breast. Some related examples where a GLRT has been employed to perform target detection include detecting buried mines from ground penetrating radar (GPR) data [29] and detecting neural activity from fMRI data [30]. The key contributions of this paper are 1) the use of a statistical model for the clutter generated by heterogeneous breast tissue; 2) the application of hypothesis testing to determine tumor presence and location in breast phantoms; and 3) an analysis of the GLRT sensitivity to errors between the assumed and actual signal.

The remainder of the paper is organized in five sections. In Section II we present the assumed model for the data and formulate a GLRT for the problem of UWB microwave breast cancer detection. In Section III we describe the numerical and experimental breast phantoms that are used to evaluate the capability of the GLRT to detect tumors from microwave backscatter. The procedures used to obtain signal templates and the clutter covariance matrix used in the GLRT are given in Section IV. Sample images constructed by applying the GLRT to data obtained from several numerical and experimental breast phantoms are provided in Section V. Finally, Section VI addresses the performance of the GLRT under matched and mismatched scattering scenarios.

Lower and upper case boldface symbols denote vectors and matrices, respectively, while superscripts $T$ and -1 denote the matrix transpose and inverse, respectively.

\section{Formulation OF the Generalized LIKELIHOOD RATIO TEST}

Our multiple hypothesis testing problem for detecting malignant tumors in the breast tests a binary hypothesis for each voxel (or pixel in 2-D) of an image. When the null hypothesis is accepted, the corresponding voxel is assigned a zero value to reflect the absence of a tumor. Conversely, when the null hypothesis is rejected, a positive value is assigned to the voxel to reflect the presence of a strong scatterer such as a malignant tumor. The resulting pseudo image indicates locations of detected tumors in the breast where large voxel values suggest relatively high confidence in the decision to reject the null hypothesis, that is, declare the presence of a tumor. In this section we present a model for the data, formulate a GLRT, and describe the procedure for constructing images of detected tumors based on the GLRT.

Multichannel data is obtained via a monostatic radar paradigm in which the breast is illuminated with an UWB pulse from one antenna array element and backscatter data is recorded at the same antenna. This is repeated for $M$ antenna positions. The observation vector from channel $i=1,2, \ldots, M$ is a length- $N$ time series which may contain backscatter contributions of the following nature: antenna reverberation, reflections from the skin-breast interface, clutter due to the heterogeneous dielectric properties of normal breast tissue, backscatter from possible tumors, and noise. The first two contributions dominate the backscatter and hinder tumor detection unless they are removed. We preprocess the data with the artifact removal algorithm proposed by Bond et al. [25]. This data-adaptive algorithm is based on the assumption that the unwanted artifacts (antenna reverberation and reflection from the skin-breast interface) in all channels possess similar but not identical time evolutions. Consequently the artifact in channel $i$ can be accurately estimated and removed using a filtered combination of all other channels.

After artifact removal, the channel $i$ time series of received backscatter, $\mathbf{y}_{i}$, is assumed to contain only signal $\alpha_{\ell_{0}} \mathbf{s}_{i}\left(\boldsymbol{\theta}_{\ell_{0}}\right)$, clutter $\mathbf{c}_{i}$, and noise $\mathbf{n}_{i}$ components

$$
\mathbf{y}_{i}=\alpha_{\ell_{0}} \mathbf{s}_{i}\left(\boldsymbol{\theta}_{\ell_{0}}\right)+\mathbf{c}_{i}+\mathbf{n}_{i}
$$

where $\boldsymbol{\theta}_{\ell}, \ell=1,2, \ldots, L$ is a vector parameterization of the physical characteristics of the scattering scenario, and the index $\ell_{0}$ corresponds to the true scattering scenario for the data. The signal vector $\mathbf{s}_{i}\left(\boldsymbol{\theta}_{\ell_{0}}\right)$ denotes a normalized time series of the backscatter signal due to the scattering scenario parameterized by $\boldsymbol{\theta}_{\ell_{0}}$. If no scatterers are present then the scale factor $\alpha_{\ell_{0}}$ is zero. The parameterization of the backscattered signal defined by $\boldsymbol{\theta}_{\ell_{0}}$ may describe any relevant features of the scattering problem including the scatterer location, size, shape, and density. For ease of exposition we assume scatterer location is the sole parameter in $\boldsymbol{\theta}_{\ell_{0}}$ as we develop the GLRT in this section. That is, we assume that the tumor size, shape, density, etc., are perfectly known. In Section VI, we consider additional scattering parameters for the GLRT.

Let $\boldsymbol{\theta}_{\ell}=\mathbf{r}_{\ell}, \ell=1, \ldots, L$ where $\mathbf{r}_{\ell}$ denotes the $\ell$ th candidate tumor location from a set of $L$ locations. Then formulate a set of $L$ binary hypothesis tests where the null hypothesis $H_{\ell}$ asserts that no tumor is present at location $\mathbf{r}_{\ell}$, and $A_{\ell}$ is the alternative hypothesis

$$
H_{\ell}: \alpha_{\ell}=0 \text { vs. } A_{\ell}: \alpha_{\ell} \neq 0 .
$$

Each location is tested independently of all other locations. This strategy is appropriate for detecting single tumors or multiple tumors that are spatially separated, assuming negligible interaction between them. That is, in the multiple tumor case we assume scattering effects are approximately linear. This assumption is reasonable for clinical screening applications where distinguishing two tumors that occur very close together in the breast is less of a concern than detecting their presence. By testing each candidate tumor location independently, the computational expense is kept low and is proportional to the number of test locations.

Space-time column vectors for the data, signal, clutter, and noise are formed by stacking the time-series column vectors for each channel, that is $\mathbf{y}=\left[\mathbf{y}_{1}^{T} \mathbf{y}_{2}^{T} \ldots \mathbf{y}_{M}^{T}\right]^{T}$. We assume that the $N M \times 1$ deterministic signal vector $\mathbf{s}\left(\boldsymbol{\theta}_{\ell}\right)$ is perfectly known 
but the amplitude factor $\alpha_{\ell}$ is deterministic and unknown. The clutter and noise are assumed to be Gaussian distributed as $\mathbf{c}+$ $\mathbf{n} \sim \mathcal{N}\left(\mathbf{0}, \sigma^{2} \mathbf{R}\right)$ where $\mathcal{N}(a, b)$ denotes the Gaussian distribution with mean $a$ and variance $b$. We assume that the covariance structure $\mathbf{R}$ is known but the power level, $\sigma^{2}$, is unknown. This Gaussian assumption for clutter is reasonable since the clutter arises from multiple independent scattering paths within the heterogeneous breast tissue. Invoking the Central Limit Theorem [31], the sum of the scattering returns is Gaussian distributed in the limit. Then the backscatter data and the signal vectors are whitened by the following linear transformations:

$$
\begin{aligned}
\mathbf{x} & =\mathbf{R}^{-1 / 2} \mathbf{y} \\
\mathbf{u}\left(\boldsymbol{\theta}_{\ell}\right) & =\mathbf{R}^{-1 / 2} \mathbf{s}\left(\boldsymbol{\theta}_{\ell}\right) .
\end{aligned}
$$

The GLRT test statistic is given by the likelihood ratio of the two hypotheses using the maximum likelihood estimates of the unknown parameters $\sigma^{2}$ and $\alpha$. For the given data model, the likelihood ratio reduces to the ratio of the unbiased variance estimates under the null and alternative hypotheses raised to the $N M / 2$ power [28]

$$
\begin{aligned}
& \Lambda_{\ell}=\frac{p\left(\mathbf{x} ; A_{\ell}, \hat{\alpha}, \widehat{\sigma^{2}}\right)}{p\left(\mathbf{x} ; H_{\ell}, \hat{\alpha}, \widehat{\sigma^{2}}\right)} \\
&=\left(\frac{\widehat{\sigma^{2}} \mid H_{\ell}}{\widehat{\sigma^{2}} \mid A_{\ell}}\right)^{N M / 2} \\
&=\left(\frac{\mathbf{x}^{T} \mathbf{x}}{\mathbf{x}^{T} \mathbf{P}_{\ell}^{\perp} \mathbf{x}}\right)^{N M / 2} \\
& \stackrel{A_{\ell}}{\stackrel{H_{\ell}}{\gtrless}} \eta
\end{aligned}
$$

where the projection matrix $\mathbf{P}_{\ell}=$ $\mathbf{u}\left(\boldsymbol{\theta}_{\ell}\right)\left[\mathbf{u}^{T}\left(\boldsymbol{\theta}_{\ell}\right) \mathbf{u}\left(\boldsymbol{\theta}_{\ell}\right)\right]^{-1} \mathbf{u}^{T}\left(\boldsymbol{\theta}_{\ell}\right)$ projects the whitened data onto the rank-1 subspace spanned by the whitened signal vector $\mathbf{u}\left(\boldsymbol{\theta}_{\ell}\right)$, and the orthogonal projection matrix $\mathbf{P}_{\ell}^{\perp}=$ $\mathbf{I}-\mathbf{P}_{\ell}$ projects the whitened data onto the rank- $(N M-1)$ complementary subspace. It will be convenient for selecting the threshold and for analysis purposes to express (5) with the following equivalent test:

$$
T_{\ell}=(N M-1) \frac{\mathbf{x}^{T} \mathbf{P}_{\ell} \mathbf{x}}{\mathbf{x}^{T} \mathbf{P}_{\ell}^{\perp} \mathbf{x}} \underset{H_{\ell}}{\stackrel{A_{\ell}}{\gtrless}} \gamma .
$$

When the test statistic $\Lambda_{\ell}$ or $T_{\ell}$ exceeds the threshold $\eta$ or $\gamma$, respectively, the null hypothesis is rejected, that is, a scatterer is detected at location $\mathbf{r}_{\ell}$.

The thresholds $\eta$ and $\gamma$ are selected to control the false discovery rate (FDR) of the image as suggested by Benjamini and Hochberg [32]. The FDR is defined as the expected proportion of falsely rejected null hypotheses in an image which notably quantifies the error rate of the whole image rather than the error rates of each individual test. Details on calculating the threshold for a specified FDR are given in [32].

The threshold calculation for a given FDR requires that the distribution of the test statistic under the null hypothesis is known. The equivalent test given by (6) is most convenient for threshold selection since the distribution of $T_{\ell_{0}}$ is known. Under the null hypothesis $H_{\ell_{0}}$ the test statistic $T_{\ell_{0}}$ is centrally F-distributed, while under the alternative hypothesis $A_{\ell_{0}}$ it is noncentrally F-distributed with noncentrality parameter $\lambda_{\ell_{0}}$ [28]. In both cases the degrees of freedom are $\nu_{1}=1$ for the numerator and $\nu_{2}=N M-1$ for the denominator. Note that these distributions hold when the test location coincides with the actual tumor location, that is $\ell=\ell_{0}$. Performance of the GLRT under the matched case $\left(H_{\ell_{0}}, A_{\ell_{0}}\right)$ and mismatched cases $\left(H_{\ell}, A_{\ell}\right.$ where $\left.\ell \neq \ell_{0}\right)$ is discussed in Section VI.

Images of detected scatterers are constructed by performing the test (6) for a set of candidate tumor locations. For each location $\mathbf{r}_{\ell}$, the corresponding voxel value $v_{\ell}$ is assigned on a logarithmic scale as follows:

$$
v_{\ell}=\left\{\begin{array}{ll}
0, & T_{\ell} \leq \gamma \\
10 \log _{10}\left(\frac{T_{\ell}}{\gamma}\right), & T_{\ell}>\gamma
\end{array} .\right.
$$

While the test statistic and threshold do not have physical interpretations, the selection of a FDR and its corresponding threshold effectively standardizes the test so that the performance of different statistical tests with the same error rate can be directly compared [28]. Selecting a value for the FDR is arbitrary, but in general smaller values increase the threshold. We let FDR $=10^{-5}$ for all of the tests in this paper.

\section{DATA ACQUISITION}

Data for UWB radar techniques is acquired from a patient in either supine or prone position with an array of broad-band antennas placed either above or surrounding the breast, respectively. Each antenna in the array sequentially transmits an UWB microwave pulse into the breast and measures the resulting backscatter. In this paper we obtain backscatter data from numerical and experimental breast phantoms to illustrate the feasibility of detecting small tumors in the breast using the GLRT. The UWB pulses span the 1 to $11 \mathrm{GHz}$ frequency range with a spectral peak at $6 \mathrm{GHz}$ and the backscattered waveforms are pre-processed to remove the early-time artifact [25].

The simulated normal and malignant tissue properties of the phantoms are chosen to achieve a reasonable match to the dielectric properties of the corresponding tissue types. Most importantly, the dielectric contrast between the tissue simulants are chosen to mimic the contrasts observed between different biological tissues at microwave frequencies. We expect breast tissue composition to vary between patients, ranging from very fatty to extremely dense. This wide range of possible normal breast tissue compositions will result in a wide range of dielectric contrasts between malignant and normal tissue from patient to patient. We therefore consider a number of phantoms with contrasts ranging from 5:1 to represent the baseline mostly fatty breast scenario, down to less than 2:1 to represent the extremely dense breast scenario [25], [27]. We also consider heterogeneously dense scenarios in two-dimensional (2-D) numerical phantoms by increasing the variability of dielectric properties values used to represent normal breast tissue. The reduced contrast phantoms present a challenging but important scenario since there is considerable evidence to suggest that dense breast tissue is a strong risk factor for breast cancer [33]. 


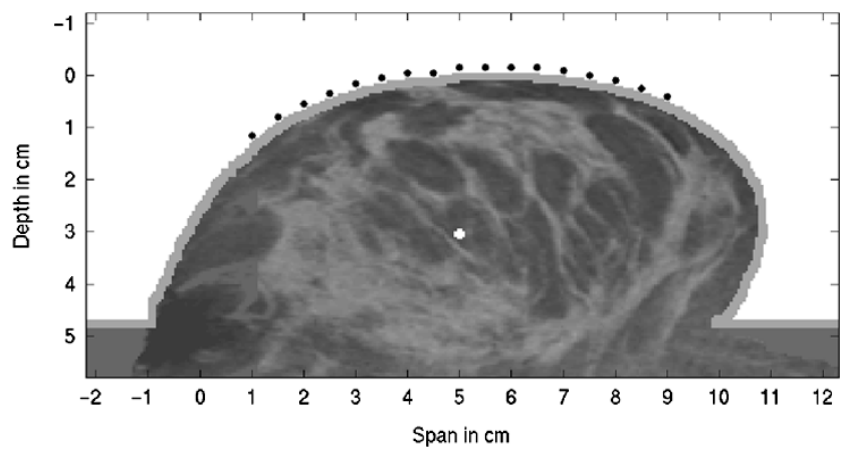

Fig. 1. Two-dimensional MRI-derived numerical model of a heterogeneously dense breast with a 2-mm-diameter tumor centered at $(5.0 \mathrm{~cm}, 3.1 \mathrm{~cm})$. The dots on the skin surface represent antenna positions. (Source: [25]).

\section{A. Two-Dimensional Numerical Breast Phantoms}

The numerical breast phantom shown in Fig. 1 is a 2-D spatial profile of dielectric properties representing the sagittal cross section of an anatomically realistic breast for a patient in the supine position [20], [25]. Dispersive properties of breast tissue are modeled using single-pole Debye models for the dielectric constant and conductivity in the phantom and the spatial variation is achieved by linearly mapping pixel intensity from a breast magnetic resonance image (MRI) to a range of dielectric properties representative of normal breast tissue [20]. A 2-mm-thick skin layer $\left(\epsilon_{r}=36.00, \sigma=4.00 \mathrm{~S} / \mathrm{m}\right)$ covers the breast and the immersion medium is matched to the dielectric properties of skin although the properties of the immersion medium have little impact in this case since the antennas are placed right on the skin surface. Cylindrical tumors like the one shown in Fig. 1 are synthetically introduced in the numerical breast phantom by increasing the dielectric properties values to represent malignant tissue, $\epsilon_{r, m}=50.73$ and $\sigma_{m}=4.82 \mathrm{~S} / \mathrm{m}$ at $6 \mathrm{GHz}$ (subscript $m$ denotes malignant breast tissue), at the desired tumor site. The tumor shown is $2 \mathrm{~mm}$ in diameter and is centered under the antenna array at a depth of $3.1 \mathrm{~cm}$. The black dots on the surface of the breast represent the elements of a conformal antenna array. The antenna elements are modeled as infinite line-sources which are sequentially excited by a 110-ps differentiated Gaussian pulse. We compute the simulated backscatter data received at each antenna using the finite-difference time-domain (FDTD) method to solve Maxwell's equations [34].

We consider a range of breast tissue compositions with ten 2-D numerical phantoms that represent various scenarios of breast tissue density and variability. Table I summarizes the average dielectric properties used for each 2-D numerical breast phantom where the normal tissue properties are varied to achieve different densities and variabilities while holding the malignant tissue properties constant. The values of the dielectric constant $\epsilon_{r, n}$ and conductivity $\sigma_{n}$ for normal breast tissue (subscript $n$ denotes normal breast tissue) range over the specified percent variation about the average value. The final column in Table I indicates the minimum malignant-to-normal dielectric contrast at $6 \mathrm{GHz}$ which ranges from the baseline case of $4.7: 1$ down to the extremely dense case of 1.18:1.
TABLE I

SUMMARY OF THE DiELECTRIC PROPERTIES FOR TEN 2-D NUMERICAL BREAST Phantoms RePRESENTING A WIDE RANGE OF NORMAL BREAST TISSUE COMPOSITIONS

\begin{tabular}{lcccc}
\hline \multicolumn{5}{c}{ Numerical breast phantoms } \\
& $\epsilon_{r, m}=50.73, \sigma_{m}=4.82$ & $\mathrm{~S} / \mathrm{m}$ at $6 \mathrm{GHz}$ \\
\hline & Average & Average & & Minimum contrast \\
$\epsilon_{r, n}$ & $\sigma_{n}(\mathrm{~S} / \mathrm{m})$ & Percent & at $6 \mathrm{GHz}$ \\
Case & at $6 \mathrm{GHz}$ & at $6 \mathrm{GHz}$ & variation & $\min \left[\frac{\epsilon_{r, m}}{\epsilon_{r, n}}\right]$ \\
\hline$n 1$ (baseline) & 9.80 & 0.40 & $\pm 10 \%$ & 4.71 \\
$n 2$ & 9.80 & 0.40 & $\pm 30 \%$ & 3.98 \\
$n 3$ & 15.65 & 1.03 & $\pm 30 \%$ & 2.49 \\
$n 4$ & 21.50 & 1.66 & $\pm 10 \%$ & 2.15 \\
$n 5$ & 21.50 & 1.66 & $\pm 20 \%$ & 1.97 \\
$n 6$ & 21.50 & 1.66 & $\pm 30 \%$ & 1.82 \\
$n 7$ & 21.50 & 1.66 & $\pm 40 \%$ & 1.69 \\
$n 8$ & 21.50 & 1.66 & $\pm 50 \%$ & 1.57 \\
$n 9$ & 27.35 & 2.29 & $\pm 30 \%$ & 1.43 \\
$n 10$ & 33.19 & 2.93 & $\pm 30 \%$ & 1.18 \\
\hline
\end{tabular}

TABLE II

SUMMARY OF THE DIELECTRIC PROPERTIES FOR THREE EXPERIMENTAL BREAST PHANTOMS REPRESENTING A RANGE OF MALIGNANT-TO-NORMAL TISSUE DIELECTRIC CONTRASTS

\begin{tabular}{lccc}
\hline \multicolumn{4}{c}{ Experimental breast phantoms } \\
$\epsilon_{r, n}=2.61, \sigma_{n}=0.05 \mathrm{~S} / \mathrm{m}$ \\
\hline Case & $\epsilon_{r, m}$ & $\sigma_{m}(\mathrm{~S} / \mathrm{m})$ \\
\hline$e 1$ (baseline) & at $6 \mathrm{GHz}$ & at $6 \mathrm{GHz}$ & Contrast at $6 \mathrm{GHz}$ \\
$e 2$ & 13.50 & 3.14 & 5.17 \\
$e 3$ & 8.65 & 1.90 & 3.31 \\
\hline
\end{tabular}

\section{B. Three-Dimensional Experimental and Numerical Breast Phantoms}

The experimental phantom setup emulates a system configuration where a patient is in a supine position with a 2-D planar antenna array placed near the surface of the naturally flattened breast [27]. The breast phantom consists of a large rectangular container filled with a liquid fat simulant $\left(\epsilon_{r, n}=2.61\right.$ and $\sigma_{n}=0.05 \mathrm{~S} / \mathrm{m}$ at $6 \mathrm{GHz}$ ), a thin dielectric slab representing skin $\left(\epsilon_{r}=4.34\right.$ with a loss tangent of 0.016 at $\left.1 \mathrm{GHz}\right)$, and a synthetic tumor suspended in the fat simulant. As noted in [27], these materials mimic the expected dielectric contrast rather than the absolute dielectric properties. The tumor is cylindrical in shape with height and diameter equal to $4 \mathrm{~mm}$ and the dielectric properties of the tumor are varied as we describe in the next paragraph. A single UWB antenna (described and characterized in [35]) is sequentially repositioned in the horizontal plane to synthesize a 2-D antenna array placed above the skin. We use the fat simulant as the immersion medium for the antenna since the current UWB antenna operates best in a high-impedance, low-loss environment.

Different contrasts between malignant and normal tissue simulants are most conveniently achieved in the experimental breast phantoms by changing the properties of the malignant tissue simulant rather than changing the normal tissue simulant as we did in the 2-D phantoms. No heterogeneity is modeled by the normal breast tissue in this experimental breast phantom, so the dielectric contrast is simply defined by the ratio $\epsilon_{r, m} / \epsilon_{r, n}$ at 6 $\mathrm{GHz}$. We consider the three experimental breast phantoms summarized in Table II with contrasts ranging from the baseline 


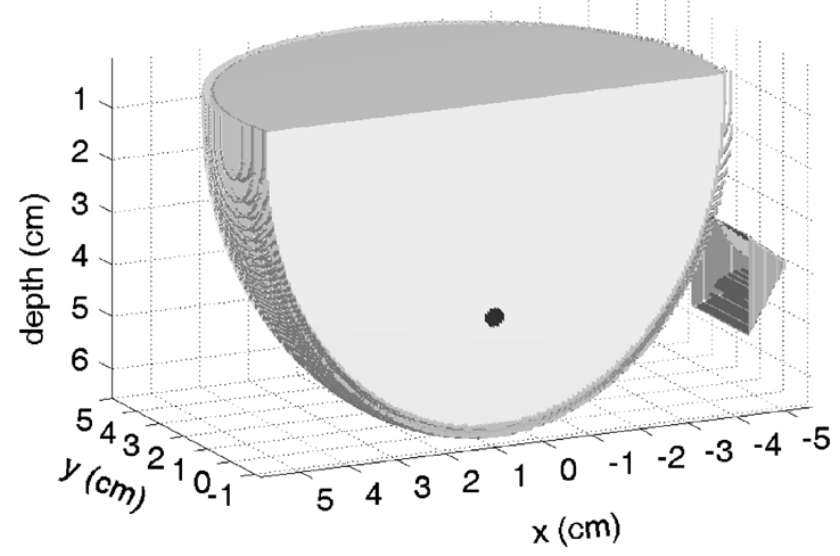

Fig. 2. Three-dimensional numerical breast phantom with realistic ellipsoidal contour. A portion of the phantom is cut away for illustration purposes, revealing a 4-mm-diameter spherical tumor in the otherwise homogeneous interior. A single UWB antenna is scanned around the pendulant breast in a circular manner at three different depths.

case of 5.17:1 down to the challenging reduced-contrast case of 1.50:1.

The UWB antenna is sequentially scanned in $1-\mathrm{cm}$ increments to 49 different positions in a $6-\mathrm{cm} \times 6-\mathrm{cm}$ array. The synthesized input pulse is a 110-ps modulated Gaussian pulse. Complete details on the experimental materials and measurement procedure are reported in [27].

In addition to the three-dimensional (3-D) experimental phantoms, we also obtain data from a 3-D numerical phantom representing a patient in the prone position as illustrated in Fig. 2. Notably, the 3-D numerical phantom differs from the experimental phantom in its realistic breast shape modeled by half of the ellipsoid $x^{2} / a^{2}+y^{2} / b^{2}+z^{2} / c^{2}=1$, where $z$ is the depth direction and we let $a=b=5 \mathrm{~cm}$ and $c=6 \mathrm{~cm}$. A layer of skin $\left(\epsilon_{r}=36.00, \sigma=4.00 \mathrm{~S} / \mathrm{m}\right)$ approximately $2 \mathrm{~mm}$ thick covers the homogeneous dispersive breast interior $\left(\epsilon_{r, n}=9.80, \sigma=0.40 \mathrm{~S} / \mathrm{m}\right.$ at $\left.6 \mathrm{GHz}\right)$. A 4-mm-diameter spherical tumor $\left(\epsilon_{r, m}=50.73, \sigma=4.82 \mathrm{~S} / \mathrm{m}\right.$ at $\left.6 \mathrm{GHz}\right)$ is introduced into the phantom at $(x=0 \mathrm{~cm}, y=-1 \mathrm{~cm}, z=4.15 \mathrm{~cm})$ as shown by the cut away depiction of the phantom. The single antenna shown in Fig. 2 is a numerical replica of our experimental UWB antenna [35]. The immersion medium assumed in the FDTD model mimics the immersion medium used in the experimental phantom $\left(\epsilon_{r}=2.61, \sigma=0.05\right)$ and as with the experimental data acquisition, the antenna is sequentially repositioned to represent an array with a total of 24 locations around the pendulant breast. We position the antenna at three depths, $2.65 \mathrm{~cm}, 4.15 \mathrm{~cm}$, and $5.65 \mathrm{~cm}$, and for each depth the antenna position is rotated around the breast in 4current $5^{\circ}$ increments.

\section{GLRT IMPLEMENTATION FOR EXAMPLE BREAST PHANTOMS}

In our formulation of the GLRT test statistic (6), we assume that the signal vectors $\mathbf{s}_{i}\left(\boldsymbol{\theta}_{\ell}\right)$ and clutter-plus-noise covariance matrix $\mathbf{R}$ are known. In this section we discuss the procedures used to obtain the signal vectors and clutter-plus-noise covariance matrix for the GLRT examples in this paper.

\section{A. Signal Vectors}

We obtain signal vector $\mathbf{s}_{i}\left(\boldsymbol{\theta}_{\ell}\right)$ analytically assuming that a single cylindrical (in 2-D) or spherical (in 3-D) scatterer is located at a specified position in an otherwise homogeneous medium. We will often refer to these analytically obtained signal vectors as signal templates. In the simple scattering scenario described, the analytical solutions for the backscatter as a function of position depend only on the scatterer diameter and the dielectric properties for the scatterer and surrounding medium. Let $\mathbf{F}=\left[\begin{array}{lllll}\epsilon_{r, n} & \sigma_{n} & \epsilon_{r, m} & \sigma_{m} & d\end{array}\right]$ be a frequencydependent vector summarizing a scattering scenario where $\epsilon_{r, n}$ and $\sigma_{n}$ are the dispersive dielectric constant and conductivity of the surrounding medium, $\epsilon_{r, m}$ and $\sigma_{m}$ are the dispersive dielectric constant and conductivity of the scatterer, and $d$ is the diameter of the scatterer. Then for an image with $L$ voxels, the set of parameter vectors $\left\{\boldsymbol{\theta}_{\ell}\right\}_{\ell=1}^{L}$ used for detection is given by

$$
\boldsymbol{\theta}_{\ell}=\left[\begin{array}{ll}
\mathbf{r}_{\ell} & \mathbf{F}
\end{array}\right]^{T}, \quad \ell=1, \ldots, L
$$

where $\mathbf{r}_{\ell}$ is the position of the $\ell$ th candidate tumor location (or equivalently the $\ell$ th voxel location). It is straightforward to include additional parameters in $\boldsymbol{\theta}_{\ell}$.

The 2-D backscatter signal templates are obtained for an infinite-length dielectric cylinder centered at $\mathbf{r}_{\ell}$, oriented parallel to an electric-current line source $I_{e}$ located at $\mathbf{r}_{i}$. The analytical expression for the backscattered electric field at sensor $i$ is given by

$$
\begin{aligned}
E_{c}\left(\boldsymbol{\theta}_{\ell}, \mathbf{r}_{i}, \omega\right) & =\frac{\omega \mu_{0} I_{e}}{4} \sum_{\nu=-\infty}^{\infty} a_{\nu} H_{\nu}^{(2)}\left(k_{n}\left|\mathbf{r}_{\ell}-\mathbf{r}_{i}\right|\right) \\
a_{\nu} & =\frac{J_{\nu}^{\prime}\left(x_{n}\right) J_{\nu}\left(x_{m}\right)-\sqrt{\frac{\epsilon_{r, m}}{\epsilon_{r, n}}} J_{\nu}\left(x_{n}\right) J_{\nu}^{\prime}\left(x_{m}\right)}{\sqrt{\frac{\epsilon_{r, m}}{\epsilon_{r, n}}} H_{\nu}^{(2)}\left(x_{n}\right) J_{\nu}^{\prime}\left(x_{m}\right)-H_{\nu}^{(2)^{\prime}}\left(x_{n}\right) J_{\nu}\left(x_{m}\right)}
\end{aligned}
$$

where $\left|\mathbf{r}_{\ell}-\mathbf{r}_{i}\right|$ is the distance between the $\ell$ th scatterer position and $i$ th antenna position, $\mu_{0}$ is the permeability of free space, $H_{\nu}^{(2)}$ denotes the $\nu$ th-order Hankel function of the second kind, and $J_{\nu}$ denotes the $\nu$ th-order Bessel function. The prime $(')$ indicates a derivative with respect to the argument of the function. The arguments of the Hankel and Bessel functions are given by $x_{n}=k_{n} d / 2$ and $x_{m}=k_{m} d / 2$, where $d$ is the diameter of the scatterer, and $k_{n}$ and $k_{m}$ are the frequencydependent wave numbers for the surrounding and scattering media, respectively.

For 3-D scattering we obtain analytical backscatter solutions for a plane wave incident on a dielectric sphere. If the plane wave propagates in the direction of $\mathbf{r}_{\ell}-\mathbf{r}_{i}$ and we model the plane wave source as a uniform sheet of current $I_{s}$ centered at 
$\mathbf{r}_{i}$ then the expression for the co-polarized component of the backscattered electric field is given by (see [36])

$$
\begin{aligned}
& E_{s}\left(\boldsymbol{\theta}_{\ell}, \mathbf{r}_{i}, \omega\right)= \frac{\omega \mu_{0} I_{s} e^{-j k_{n}\left|\mathbf{r}_{\ell}-\mathbf{r}_{i}\right|}}{4 k_{n}^{2}\left|\mathbf{r}_{\ell}-\mathbf{r}_{i}\right|} \sum_{\nu=1}^{\infty} j^{\nu-1}(2 \nu+1) \\
& \cdot\left[a_{\nu} \hat{H}_{\nu}^{(2)^{\prime}}\left(k_{n}\left|\mathbf{r}_{\ell}-\mathbf{r}_{i}\right|\right)+j b_{\nu} \hat{H}_{\nu}^{(2)}\left(k_{n}\left|\mathbf{r}_{\ell}-\mathbf{r}_{i}\right|\right)\right] \\
& a_{\nu}= \frac{\hat{J}_{\nu}\left(x_{n}\right) \hat{J}_{\nu}^{\prime}\left(x_{m}\right)-\sqrt{\frac{\epsilon_{r, m}}{\epsilon_{r, n}}}}{\hat{J}_{\nu}\left(x_{m}\right) \hat{J}_{\nu}^{\prime}\left(x_{n}\right)} \\
& \hat{H}_{\nu}^{(2)}\left(x_{n}\right) \hat{J}_{\nu}^{\prime}\left(x_{m}\right)-\sqrt{\frac{\epsilon_{r, m}}{\epsilon_{r, n}}} \hat{J}_{\nu}\left(x_{m}\right) \hat{H}_{\nu}^{(2)^{\prime}}\left(x_{n}\right) \\
& b_{\nu}= \frac{\sqrt{\frac{\epsilon_{r, m}}{\epsilon_{r, n}}}}{\sqrt{\frac{\epsilon_{r, m}}{\epsilon_{r, n}}}} \hat{H}_{\nu}^{(2)}\left(x_{n}\right) \hat{J}_{\nu}^{\prime}\left(x_{m}\right)-\hat{J}_{\nu}\left(x_{m}\right) \hat{J}_{\nu}^{\prime}\left(x_{n}\right) \\
&
\end{aligned}
$$

where $\hat{H}_{\nu}^{(2)}(x)=\sqrt{\pi x / 2} H_{\nu+1 / 2}^{(2)}(x)$ is the $\nu$ th-order spherical Hankel function of the second kind and $\hat{J}_{\nu}(x)=$ $\sqrt{\pi x / 2} J_{\nu+1 / 2}(x)$ is the $\nu$ th-order spherical Bessel function.

In the 3-D numerical phantom, the antennas are immersed in a medium with dielectric properties that differ from those of normal breast tissue. Thus the propagating plane wave travels through multiple media, a fact which should be accounted for in the signal templates. In the 3-D numerical phantom, we use (10) to model the propagation of a plane wave in normal breast tissue and the scattering effects of the tumor. Then we separately model the propagation effects of a plane wave in the immersion and skin media for the respective round-trip distances traveled. The composite propagation model is obtained by multiplying the three frequency-domain propagation models for the three media. This analytical solution neglects any reflections at the immersion medium-skin and skin-breast interfaces. Since we are assuming plane waves normally incident on planar interfaces and since we are not concerned with signal amplitudes (recall that we estimate the signal amplitude from the data in the GLRT) we expect this simplified propagation model to be sufficient.

The 2-D numerical and 3-D experimental setups also involve propagation through multiple media, but for these phantoms we approximate the propagation effects with a single homogeneous medium since the propagation path is predominantly through the breast medium. For the 2-D numerical case this is true because the antennas are located on the surface of the thin skin layer so the immersion medium has negligible effect on the fields that propagate into the breast. This is also true for the 3-D experimental case since the immersion medium and the fat simulant are the same and have identical propagation effects.

Time-domain signal templates $\mathbf{s}_{i}\left(\boldsymbol{\theta}_{\ell}\right)$ are obtained from the propagation and scattering models described in this section by performing an inverse discrete Fourier transform (IDFT) on the electric field for a set of DFT frequencies spanning 1-11 GHz.

\section{B. Clutter Covariance Matrix}

Our formulation of the GLRT in Section II assumes that the clutter-plus-noise component of the backscattered signal is Gaussian distributed with covariance matrix $\sigma^{2} \mathbf{R}$. Let $\mathbf{R}=\mathbf{R}_{c}+\mathbf{R}_{v}$ be a decomposition of the clutter covariance matrix into clutter and noise covariance matrices, respectively. We assume that the noise component is white, that is $\mathbf{R}_{v}=\sigma_{v}^{2} \mathbf{I}$. For the 3-D experimental and numerical phantoms, the normal breast tissue is homogeneous and will not produce any significant random scattering or clutter in the backscatter data, so we model only the white Gaussian noise component in these cases. That is, $\mathbf{R}=\mathbf{R}_{v}$ for the 3-D breast phantoms. The 2-D MRI-derived numerical breast phantoms, however, do produce a random clutter component in the backscatter due to the variability of the normal breast tissue composition from one patient to the next. In the following discussion we describe the method used to model the clutter covariance matrix for the 2-D numerical breast phantoms.

The clutter is completely characterized by its covariance matrix since it is assumed to be zero-mean Gaussian distributed. The full space-time clutter covariance matrix has dimensions $N M \times N M$ and approximately $(N M)^{2} / 2$ free parameters. ${ }^{1}$ For typical values of $N$ and $M(N \sim 100, M \sim 20)$ the number of parameters in the full space-time clutter matrix is far too large for reliable estimation in practical scenarios. Hence, we generate a simplified space-time clutter covariance matrix model by assuming: (1) the clutter observed in channel $i$ is independent of the clutter observed in channel $j$ for $i \neq j$, and (2) the clutter observed in channel $i$ is distributed identically to the clutter in channel $j$. The first assumption relies on the presence of attenuation, which causes the clutter in each channel to be dominated by heterogeneity in the immediate vicinity of the antenna. The second assumption requires that the degree of heterogeneity in the vicinity of each antenna be similar. Thus, the covariance matrix is assumed to take on a block diagonal form with identical blocks representing the temporal covariance of the clutter. With these assumptions, only the $N \times N$ temporal covariance matrix $\mathbf{R}_{t}$ must be estimated. We estimate $\mathbf{R}_{t}$ via the sample covariance matrix constructed using $K$ observations of the $N \times 1$ clutter time-series $\mathbf{c}_{i}$ as follows:

$$
\hat{\mathbf{R}}_{t}=\frac{1}{K} \sum_{i=1}^{K} \mathbf{c}_{i} \mathbf{c}_{i}^{T} .
$$

The space-time clutter covariance matrix is thus

$$
\hat{\mathbf{R}}_{c}=\left[\begin{array}{llll}
\hat{\mathbf{R}}_{t} & & & \\
& \hat{\mathbf{R}}_{t} & & \\
& & \ddots & \\
& & & \hat{\mathbf{R}}_{t}
\end{array}\right] .
$$

The inverse square-root of the clutter-plus-noise covariance matrix $\mathbf{R}^{-1 / 2}=\left(\mathbf{R}_{c}+\sigma_{v}^{2} \mathbf{I}\right)^{-1 / 2}$ is a whitening operator for the backscatter data in the GLRT. The matrix inversion of $\mathbf{R}$ is well-defined even for singular $\mathbf{R}_{c}$ since the noise covariance term acts at a regularization parameter.

For each of the 2-D numerical phantoms listed in Table I we estimate a temporal clutter covariance matrix from $K=306$ clutter observations obtained from 18 MRI-based FDTD breast phantoms similar to the one shown in Fig. 1. To isolate the clutter component in the backscatter waveforms, we subtract

\footnotetext{
${ }^{1}$ Note that the clutter in any channel is not wide-sense stationary due to atten-
} uation in the breast, so the temporal covariance matrix is not Toeplitz. 
the skin reflection using the corresponding backscatter waveforms from an internally homogeneous breast phantom. The breast phantoms used to generate clutter observations are all tumor-free and each phantom has a unique tissue structure. The 18 distinct tissue structures are obtained from 6 well separated sagittal slices selected from the 3-D MRI's of 3 patients. We obtain 17 independent clutter time-series observations, one at each of the 17 sensors in the conformal antenna array, for a total of $K=306$ clutter time series for each of the phantoms described in Table I. The corresponding clutter covariance matrices are estimated for each phantom using (11) and (12).

Assuming that the energy of the additive white noise is much lower than the clutter energy we let $\sigma_{v}^{2} \approx$ $((1) /(100 N M)) \operatorname{trace}\left[\widehat{\mathbf{R}_{c}}\right]$.

\section{RESULTS}

In this section we present several example images obtained by applying the GLRT to backscatter from the numerical and experimental breast phantoms described in Section III. We introduce very small $(\leq 0.6 \mathrm{~cm})$ synthetic tumors into the breast phantoms to represent the small growths of early stage breast cancer.

\section{A. GLRT-Derived Images for 2-D Numerical Breast Phantoms}

For the examples presented in this subsection, we have applied the GLRT to backscatter data obtained from several 2-D numerical breast phantoms with dielectric properties described in Table I. The phantoms may have different tumor configurations, but they are all introduced onto the same MRI-derived normal breast tissue architecture. The corresponding GLRT's are constructed using signal templates parameterized to match the average scattering properties of the appropriate breast phantom. That is, we obtain the analytical signal templates for cylindrical scatterers of the same diameter as the tumors in the breast phantoms, matching the dielectric properties for the background and scatterer media to the average values for normal and malignant tissues of the phantoms in Table I. The signal templates are further parameterized by the position $\mathbf{r}_{\ell}$ of the tumor which is scanned over a $10 \mathrm{~cm} \times 4 \mathrm{~cm}$ region of the breast with $1 \mathrm{~mm}^{2}$ spatial sampling.

Fig. 3(a) depicts a GLRT-derived image of detected scatterers without clutter whitening, that is $\mathbf{R}=\mathbf{I}$, for backscatter from an $n 6$ breast phantom with a single 2-mm-diameter tumor centered at $(\mathrm{span}$, depth $)=(5.0 \mathrm{~cm}, 2.1 \mathrm{~cm})$. The $n 6$ phantom was chosen because of the relatively wide range of variability in this phantom which introduces a significant clutter component into the backscatter data. Pixels with positive values of the test statistic indicate locations where the null hypothesis is rejected, implying that a scatterer has been detected. The solid black contour lines delineate the threshold. The resulting GLRT image in Fig. 3(a) is littered with false detections due to the clutter backscatter in the data, particularly near the surface of the breast and in the lower left hand corner. The peak test statistic occurs at $(5.0 \mathrm{~cm}, 2.1 \mathrm{~cm})$ with a peak value $8.9 \mathrm{~dB}$ above the threshold. Although this peak correctly detects and localizes the true tumor in the breast phantom, the next largest peak in the image occurs at $(0.4 \mathrm{~cm}, 3.6 \mathrm{~cm})$ with a value $5.8 \mathrm{~dB}$ above the threshold. This

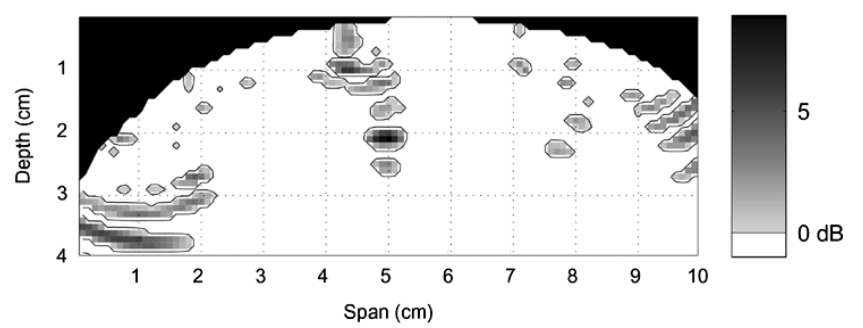

(a)

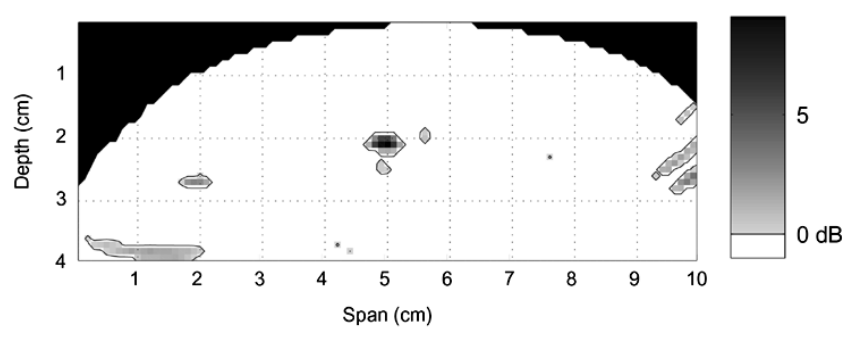

(b)

Fig. 3. Detection images for simulated backscatter data from an $n 6$ numerical breast phantom similar to the one shown in Fig. 1. A single 2-mm-diameter tumor is centered at $(5.0 \mathrm{~cm}, 2.1 \mathrm{~cm})$. The image depicted in (a) was created by a GLRT that did not use clutter whitening, while the image shown in (b) was created from a GLRT incorporating clutter whitening.

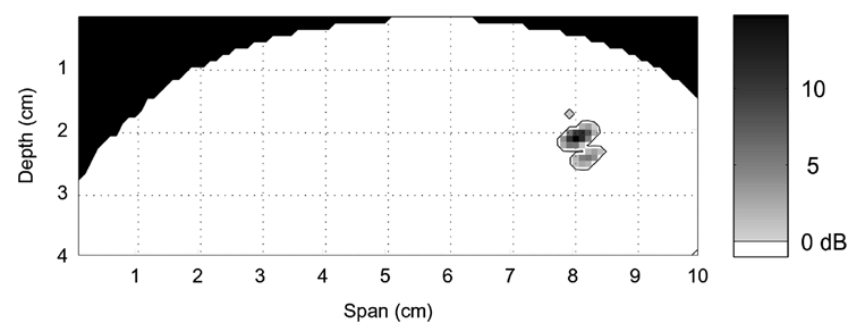

Fig. 4. Detection image for simulated backscatter data from an $n 1$ numerical breast phantom with a single $2-\mathrm{mm}$-diameter tumor centered at $(8.0 \mathrm{~cm}, 2.1 \mathrm{~cm})$.

detected region, along with several other regions of false detections throughout the image, is due to the clutter backscatter. In contrast, Fig. 3(b) depicts the corresponding GLRT-derived image when the clutter covariance matrix of (12) is incorporated into the GLRT. The false detections are greatly reduced in comparison to Fig. 3(a) and the detected scatterer at $(5.0 \mathrm{~cm}, 2.1$ $\mathrm{cm}$ ), which coincides with the true tumor location, dominates the image with a peak value $9.1 \mathrm{~dB}$ above the threshold. The few remaining regions of falsely detected scatterers in Fig. 3(b) are due to clutter, but the test statistics in these regions have relatively low values of $3.7 \mathrm{~dB}$ and below. These low-level false detections due to clutter are also detected for the corresponding tumor-free phantom.

Fig. 4 depicts a detection image for a breast phantom exhibiting the baseline contrast ( $n 1$ in Table I). For this phantom the single 2-mm-diameter tumor is placed off axis at $(8.0 \mathrm{~cm}$, $2.1 \mathrm{~cm}$ ). The location of the peak test statistic in the image coincides exactly with the center of the modeled tumor and the peak value is $14.8 \mathrm{~dB}$ above the threshold. Note that the peak value is much higher for this phantom than for the $n 6$ phantom due to the difference in dielectric contrast for the two phantoms. Smaller peaks or "sidelobes" of the signal occur in this image just above and below the detected scatterer, but the pixel values 


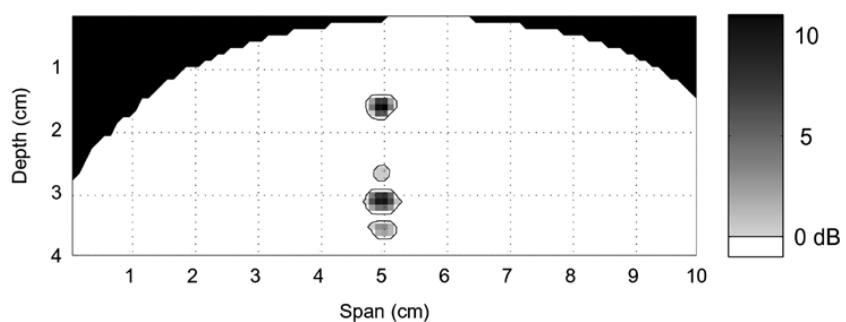

Fig. 5. Detection image for simulated backscatter data from an $n 1$ numerical breast phantom with two tumors vertically separated by $1.5 \mathrm{~cm}$. The lower tumor is centered at $(5.0 \mathrm{~cm}, 3.1 \mathrm{~cm})$.

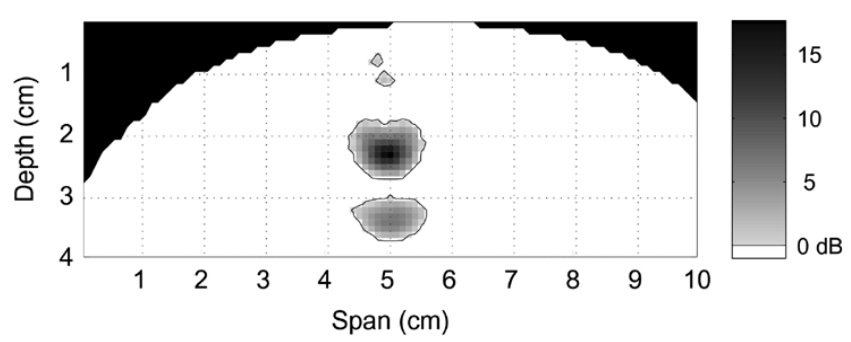

Fig. 6. Detection image for simulated backscatter data from an $n 1$ numerical breast phantom with a 6 - $\mathrm{mm}$-diameter tumor centered at $(5.0 \mathrm{~cm}, 2.3 \mathrm{~cm})$.

in the sidelobes are within a few $\mathrm{dB}$ of the threshold value. Note that false detections due to clutter have been successfully suppressed in this image.

The GLRT is also capable of detecting multifocal tumors as shown in Fig. 5. For this example the backscatter data is collected from an $n 1$ breast phantom containing two 2-mm-diameter tumors that are vertically aligned and centered at $(5.0 \mathrm{~cm}$, $1.6 \mathrm{~cm})$ and $(5.0 \mathrm{~cm}, 3.1 \mathrm{~cm})$. Two dominant peaks are apparent in the GLRT image corresponding to the two tumors in the breast phantom. The locations of the two dominant peaks in the detection image coincide exactly with the true centers of the tumors in the phantom and the peak values are $11.0 \mathrm{~dB}$ for the shallow tumor and $10.3 \mathrm{~dB}$ for the deep tumor. Again, the clutter response has been suppressed below the threshold throughout the imaging region and only low-level sidelobes are present above and below one of the detected scatterers.

In the next example, we modify the GLRT for a larger diameter tumor, $d=6 \mathrm{~mm}$, and obtain backscatter from an $n 1$ breast phantom containing a 6-mm-diameter tumor centered at $(5.0 \mathrm{~cm}, 2.3 \mathrm{~cm})$. Fig. 6 depicts the resulting detection image. The global peak occurs exactly at the center of the modeled tumor with a peak value of $17.7 \mathrm{~dB}$. Since the backscatter signal from the larger diameter tumor has greater magnitude than before, the mainlobe of the detected scatterer encompasses a larger region and more pixels around the peak exceed the threshold. The sidelobe that occurs below the detected scatterer is deeper than it was for the 2-mm-diameter tumors, but it is still relatively low level. We show in Section VI that the sidelobes occur at predictable locations. The two small low-level regions that are detected just above the main peak are most likely due to a combination of the clutter remnants after whitening and the upper sidelobe of the detected scatterer.

These examples illustrate the capability of the GLRT to detect and localize small scatterers in various configurations when

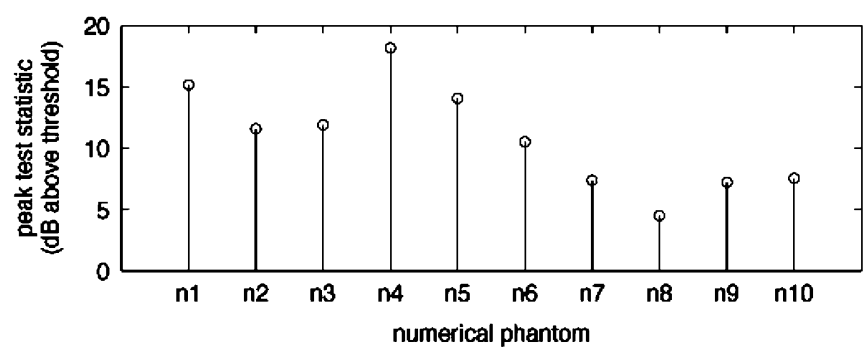

(a)

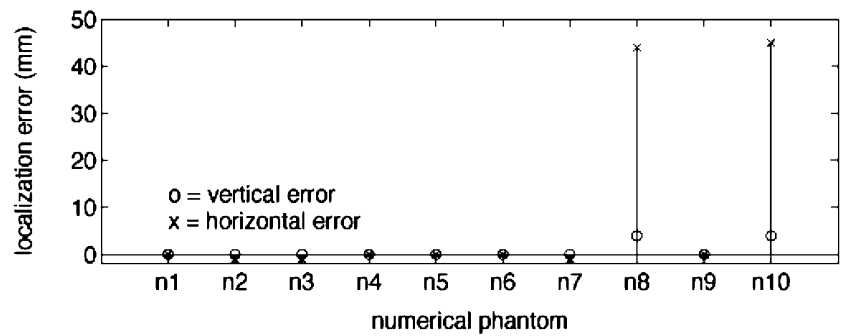

(b)

Fig. 7. Summary of GLRT results for 2-D numerical breast phantoms $n 1$ through $n 10$ (described in Table I) with a single 2-mm-diameter tumor centered at $(5.0 \mathrm{~cm}, 2.1 \mathrm{~cm})$. (a) Peak test statistic value and (b) localization error in the vertical (Depth) and horizontal (Span) directions for each case.

the scatterers exhibit the baseline contrast of $4.71: 1$ or the reduced-contrast case of 1.82:1. The examples also demonstrate that the assumed Gaussian clutter model is effective at suppressing clutter throughout the image when incorporated into the GLRT. We observe that backscatter from larger tumors results in slightly increased test statistic values compared to the backscatter from smaller tumors, and similarly, shallow tumors have higher test statistic values than deep tumors. However, regardless of position, size, or number, all of the tumors in these examples are clearly detected by the GLRT with the chosen FDR.

Several more challenging imaging scenarios are presented for the reduced contrast numerical breast phantoms listed in Table I. The results of applying the GLRT to the baseline and the reduced-contrast numerical breast phantoms are summarized by the two plots of Fig. 7. In each case the numerical breast phantom contains a single 2-mm-diameter tumor placed at $(5.0 \mathrm{~cm}, 2.1 \mathrm{~cm})$. Fig. 7(a) indicates the values of the peak test statistic in the GLRT images and Fig. 7(b) indicates the localization error in both the vertical and horizontal directions for each of the numerical breast phantoms described in Table I. For cases $n 1$ through $n 7$, where the minimum contrast is 1.69:1 or greater, the tumor is detected and localized within $2 \mathrm{~mm}$ of its true location. Case $n 9$ is also correctly detected and localized within $1 \mathrm{~mm}$ of its true location even though the minimum contrast for this phantom is only 1.43:1. In cases $n 8$ and $n 10$ which have contrasts below 1.69:1, the peak test statistic location occurs several $\mathrm{cm}$ away from the true tumor location. In both of these cases the peak occurs on the far right side of the image where a relatively high-contrast interface is formed in the numerical phantom by the spatially varying dielectric properties near the antenna array. Thus this false detection is due to a particularly large clutter response analogous to those evident on the far right side of the detection images shown in 
Fig. 3(a) and (b). Phantoms $n 8$ and $n 10$ represent very low contrast scenarios corresponding to the challenging case of heterogeneously dense breast tissue. The ratio of signal to clutter power in the backscatter signal for these cases appears to be so low that the underlying signal component cannot be detected by the GLRT. Further work and improvement on the clutter model and GLRT is needed to determine whether successful detection is possible for the heterogeneously dense cases of $n 8$ and $n 10$. However, for all of the other challenging reduced-contrast cases that we have considered here, our results suggest that the GLRT using the sample clutter covariance matrix shows promise for detecting very small tumors and suppressing clutter even when the dielectric contrast is as low as 1.43:1.

\section{B. GLRT-Derived Images for 3-D Breast Phantoms}

Next, we consider the GLRT applied to backscatter waveforms from the 3-D experimental and numerical breast phantoms. The GLRT's for the 3-D phantoms are constructed using analytical signal templates for a 4-mm-diameter spherical tumor where the dielectric properties of the scatterer and surrounding medium match the corresponding dielectric properties of the 3-D phantoms. Note that in the experimental case the scatterer shape does not perfectly match the scatterer shape assumed in the GLRT templates. We allow this design mismatch because the cylindrical shape is most convenient for constructing the experimental phantom and the spherical shape leads to a tractable analytical solution for the templates. We expect the impact of the mismatch to be minor since the dimensions of the cylinder and sphere are comparable and the tumor size is smaller than the wavelength at the center frequency of the UWB pulse. In addition to scattering from the spherical tumor, the templates for the 3-D numerical phantom account for propagation through multiple layers: the immersion medium, skin, and breast tissue. The distance from each antenna position to the skin is estimated (in the direction of the incident field) and the thickness of the skin is assumed to be $2 \mathrm{~mm}$. These imperfectly approximated distances as well as the curvature of the skin contour and the radiation pattern of the antenna will lead to some practical mismatches between the actual and assumed signals for the 3-D numerical phantom. The signal templates are further parameterized by the position $\mathbf{r}_{\ell}$ of the tumor which is scanned with $1 \mathrm{~mm}^{2}$ spatial resolution over a region of the breast phantom. For the experimental phantoms, this imaging region is the $6 \mathrm{~cm} \times 6 \mathrm{~cm} \times 5 \mathrm{~cm}$ region of the breast phantom directly below the antenna array. For the 3 -D numerical phantom, the imaging region is $8 \mathrm{~cm} \times 8 \mathrm{~cm} \times 5.6 \mathrm{~cm}$ and centered within the circular antenna array.

We first present results for the experimental breast phantom with the intermediate contrast ( $e 2$ of Table II). We obtain experimental backscatter data from a phantom containing a 4-mm-diameter, 4-mm-tall cylindrical scatterer located approximately at $(x$-span, $y$-span, depth $)=(0 \mathrm{~cm}, 0 \mathrm{~cm}, 2.2 \mathrm{~cm})$. Fig. 8 depicts the resulting 3-D plot of the thresholded test statistic, where a portion of the image has been cut away to reveal the spatial profile of the test statistic in the detected regions. The peak value is $16.6 \mathrm{~dB}$ above the threshold and occurs within $3 \mathrm{~mm}$ of the true tumor location. Although the tumor is well-localized by the peak voxel in this image, multiple sidelobes above and below

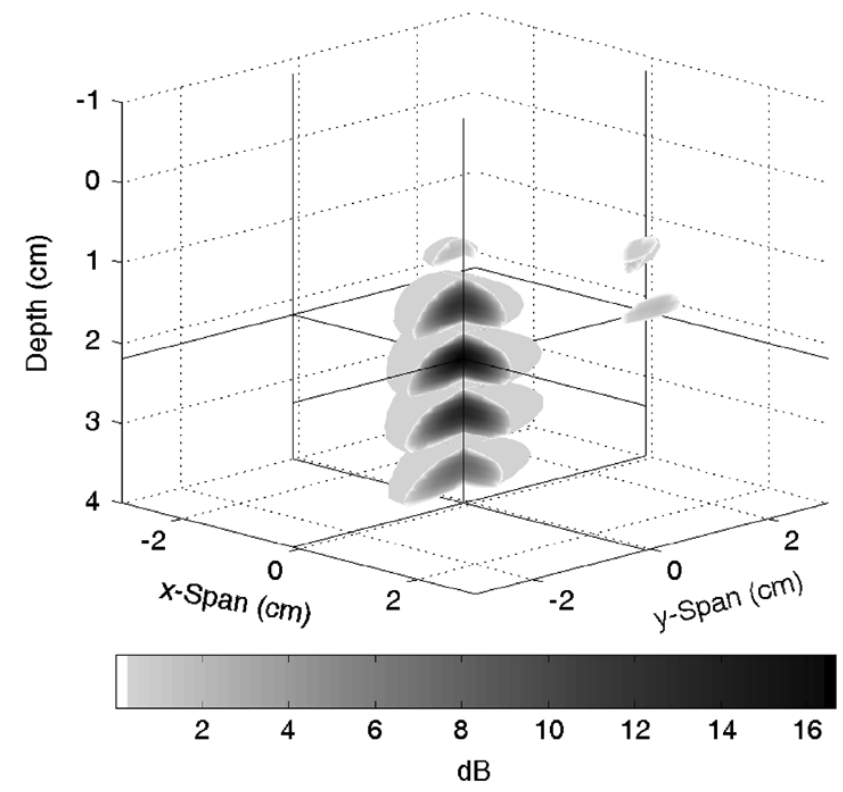

Fig. 8. Detection image for experimental backscatter data from an $e 2$ experimental breast phantom with a single $4 \mathrm{~mm}$ cylindrical tumor centered approximately at $(0 \mathrm{~cm}, 0 \mathrm{~cm}, 2.2 \mathrm{~cm})$.

the true tumor location create some ambiguity in determining the number of scatterers detected. The sidelobes are due to high correlation between the signal template at the peak voxel and the signal templates within the main and sidelobe voxels. This high correlation occurs because of the low loss and permittivity of the fat simulant in the experimental phantom and also because of the oscillating peaks of the modulated Gaussian pulse. Due to the low dielectric properties, the electrical size of the phantom and the distance between testing locations is effectively reduced for the given bandwidth. As a result, the signals are correlated for larger spatial regions than in the numerical breast phantoms. The multiple sidelobes occur due to the oscillating nature of the transmitted pulse.

Fig. 9 summarizes the results of detection for each of the 3-D experimental phantoms in Table II with a single $4 \mathrm{~mm}$ tumor approximately centered below the antenna array at a depth of $2.2 \mathrm{~cm}$. In each case a scatterer is correctly detected and localized within a few mm of the tumor location, and the peak test statistic values are all well above the threshold.

The GLRT image shown in Fig. 10 is constructed from data obtained with the 3-D numerical breast phantom. The peak test statistic is $2 \mathrm{~dB}$ above the threshold and is localized within $2 \mathrm{~mm}$ of the true tumor location. Note that the sidelobes seen in Fig. 8 are not present in this image because the more realistic dielectric properties used for the fat simulant result in greater loss and consequently lower spatial correlation in the signal templates.

\section{GLRT PERFORMANCE ANALYSIS}

In this section, we investigate the loss in GLRT detection performance as mismatch between the actual and assumed signal parameterizations is systematically introduced. Mismatch, that is $\boldsymbol{\theta}_{\ell} \neq \boldsymbol{\theta}_{\ell_{0}}$, occurs as a result of imperfect assumptions in the GLRT formulation. For example, the signal templates in the GLRT are constructed using assumed values for the average 


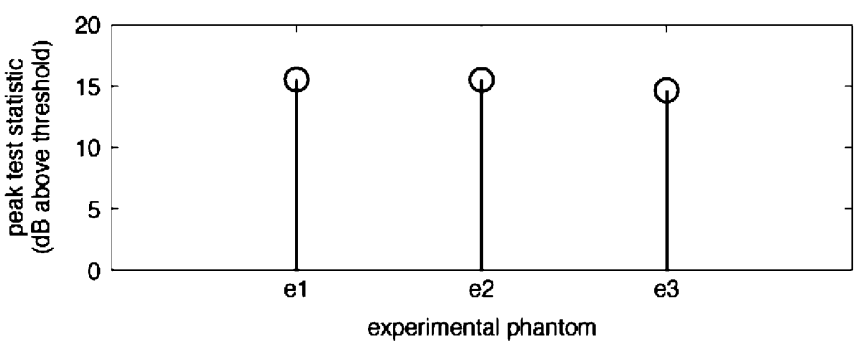

(a)

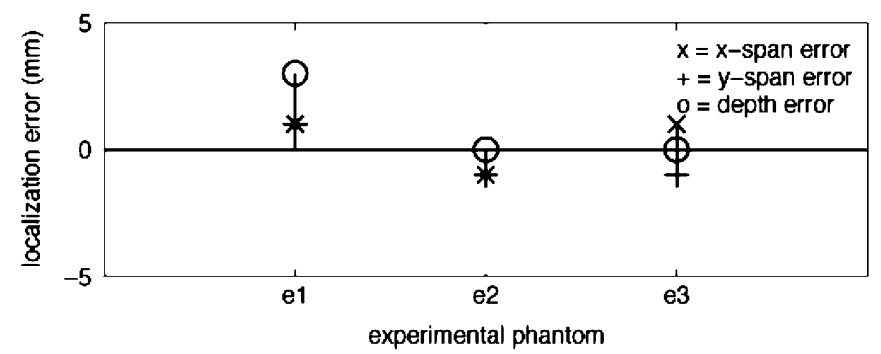

(b)

Fig. 9. Summary of GLRT results for 3-D experimental breast phantoms $e 1$ through $e 3$ (described in Table II). (a) Peak test statistic value and (b) localization error in the $x$-span, $y$-span, and depth directions for each case.

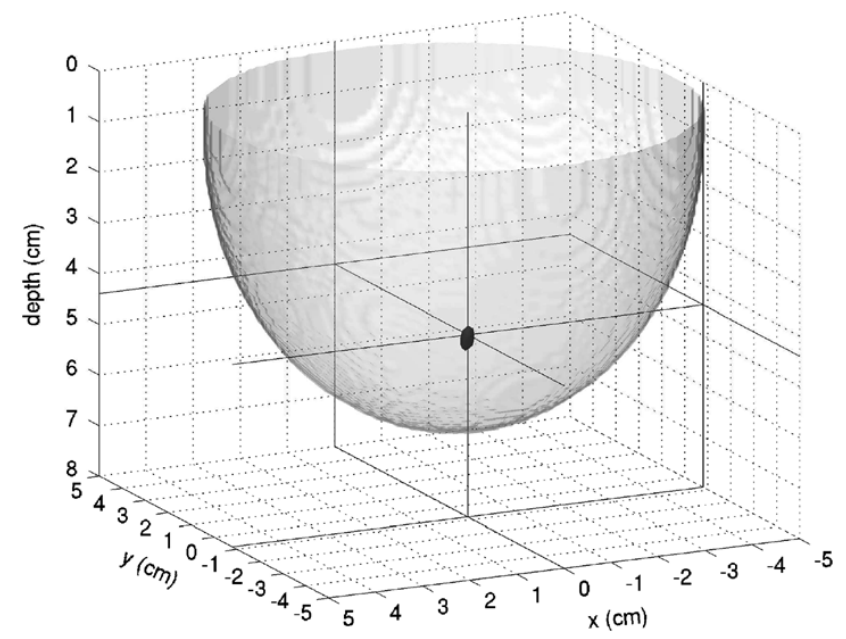

Fig. 10. Detection image for simulated backscatter data from the 3-D numerical breast phantom of Fig. 2. The transparent breast contour is superimposed on the detection image to illustrate the relative location of the detected scatterer.

dielectric properties of normal and malignant breast tissue. In reality the dielectric properties are spatially dependent within the breast and may vary considerably from patient-to-patient, so even if a good estimate of the average dielectric properties is available, it is still very likely that some spatially-dependent mismatch in dielectric properties will occur. Other likely sources of mismatch include the shape, size, contrast and location of a tumor in the breast. Thus it is important to understand the performance of the GLRT under mild to severe mismatch conditions. We begin by showing that mismatch effectively lowers the signal-to-noise ratio (SNR) of the data and that the loss in SNR is characterized by the geometric angle between the actual and assumed signal vectors. Next, we explore the relationship between GLRT power (the probability of correctly detecting a scatterer) and our expression for mismatch loss. We conclude the section with several examples of mismatch using the 2-D signal templates described Section IV-A. Throughout this section we assume for simplicity that the data vector, $\mathbf{x}$, is distributed as $\mathcal{N}\left(\alpha_{\ell_{0}} \mathbf{u}\left(\boldsymbol{\theta}_{\ell_{0}}\right), \sigma^{2} \mathbf{I}_{N M}\right)$ (that is, we assume a single scatterer exists at $\mathbf{r}_{\ell_{0}}$ and the clutter whitening is perfect).

For the test statistic given by (6), both the numerator and denominator have $\chi^{2}$ distributions and are independent of one another since the projection matrices $\mathbf{P}_{\ell}$ and $\mathbf{P}_{\ell}^{\perp}$ are mutually orthogonal. The ratio is thus singly-noncentral $F$-distributed $T_{\ell_{0}} \sim F_{1, N M-1}^{\prime}\left(\lambda_{\ell_{0}}\right)$ in the matched case $\left(\ell=\ell_{0}\right)$ and doublynoncentral $F$-distributed $T_{\ell} \sim F_{1, N M-1}^{\prime \prime}\left(\lambda_{\ell}, \lambda_{\ell}^{\perp}\right)$ under mismatched conditions $\left(\ell \neq \ell_{0}\right)$. The noncentrality parameters for these distributions are given by

$$
\begin{aligned}
\lambda_{\ell_{0}} & =\frac{\alpha_{\ell_{0}}^{2}}{\sigma^{2}} \mathbf{u}^{T}\left(\boldsymbol{\theta}_{\ell_{0}}\right) \mathbf{u}\left(\boldsymbol{\theta}_{\ell_{0}}\right) \\
\lambda_{\ell} & =\lambda_{\ell_{0}} \cos ^{2} \phi \\
\lambda_{\ell}^{\perp} & =\lambda_{\ell_{0}} \sin ^{2} \phi
\end{aligned}
$$

where

$$
\cos ^{2} \phi=\frac{\left|\mathbf{u}\left(\boldsymbol{\theta}_{\ell}\right)^{T} \mathbf{u}\left(\boldsymbol{\theta}_{\ell_{0}}\right)\right|^{2}}{\mathbf{u}\left(\boldsymbol{\theta}_{\ell}\right)^{T} \mathbf{u}\left(\boldsymbol{\theta}_{\ell}\right) \mathbf{u}\left(\boldsymbol{\theta}_{\ell_{0}}\right)^{T} \mathbf{u}\left(\boldsymbol{\theta}_{\ell_{0}}\right)} .
$$

Here, $\phi$ represents the geometric angle between assumed and actual signal vectors, $\mathbf{u}\left(\boldsymbol{\theta}_{\ell}\right)$ and $\mathbf{u}\left(\boldsymbol{\theta}_{\ell_{0}}\right)$, respectively. As $|\phi|$ increases on $[0,(\pi / 2)]$, the numerator noncentrality parameter $\lambda_{\ell}$ decreases and the denominator noncentrality parameter $\lambda_{\ell}^{\perp}$ increases, leading to a reduced test statistic in the mismatched case compared to the matched case. Note that the sum of the numerator and denominator noncentrality parameters equals $\lambda_{\ell_{0}}$, which represents the SNR for the data. We define a new variable $\lambda_{m m}=-10 \log _{10} \cos ^{2} \phi$ to denote the loss in the numerator noncentrality parameter on a logarithmic scale.

For a given $\lambda_{\ell_{0}}$ and $\phi$, the probability of correctly detecting a scatterer using the GLRT is given by

$$
P_{D}\left(\lambda_{\ell_{0}}, \phi\right)=\int_{\gamma}^{\infty} \mathrm{d} F_{1, N M-1}^{\prime \prime}\left(\lambda_{\ell_{0}} \cos ^{2} \phi, \lambda_{\ell_{0}} \sin ^{2} \phi\right)
$$

where $F_{\nu_{1}, \nu_{2}}^{\prime \prime}\left(\delta_{1}, \delta_{2}\right)$ is the doubly noncentral F cdf with $\nu_{1}$ and $\nu_{2}$ numerator and denominator degrees of freedom, respectively, and $\delta_{1}$ and $\delta_{2}$ numerator and denominator noncentrality parameters, respectively. The doubly noncentral F distribution has complex dependence on four parameters making it difficult to intuit the effects of one or more changes in the parameter values. Therefore we approximate $F_{\nu_{1}, \nu_{2}}^{\prime \prime}\left(\delta_{1}, \delta_{2}\right)$ by the singly noncentral $\mathrm{F}$ distribution $\left(1+\left(\delta_{2} / \nu_{2}\right)\right)^{-1} F_{\nu_{1}, \nu^{\prime}}^{\prime}\left(\delta_{1}\right)$ where $\nu^{\prime}=\left(\left(\nu_{2}+\right.\right.$ $\left.\left.\delta_{2}\right)^{2}\right) /\left(\nu_{2}+2 \delta_{2}\right)$ [37] to facilitate the performance analysis of the mismatched GLRT. Now both the matched $\left(\lambda_{m m}=0\right)$ and mismatched $\left(\lambda_{m m}>0\right)$ GLRT's are described by

$$
T_{\ell} \sim\left(1+\frac{\lambda_{\ell_{0}} \sin ^{2} \phi}{N M-1}\right)^{-1} F_{1, \nu^{\prime}}^{\prime}\left(\lambda_{\ell_{0}} \cos ^{2} \phi\right)
$$

where $\nu^{\prime}=\left(\left(N M-1+\lambda_{\ell_{0}} \sin ^{2} \phi\right)^{2}\right) /\left(N M-1+2 \lambda_{\ell_{0}} \sin ^{2} \phi\right)$. As $\nu^{\prime} \rightarrow \infty$, the noncentral $\mathrm{F}$ distribution approaches a noncentral $\chi^{2}$ distribution with one degree of freedom [37]. Noting that $\nu^{\prime}>(N M-1) / 2$, the noncentral $\chi^{2}$ approximation is valid for $N M$ sufficiently large, that is $\left(1+\left(\lambda_{\ell_{0}} \sin ^{2} \phi\right) /(N M-1)\right) T_{\ell} \sim$ 


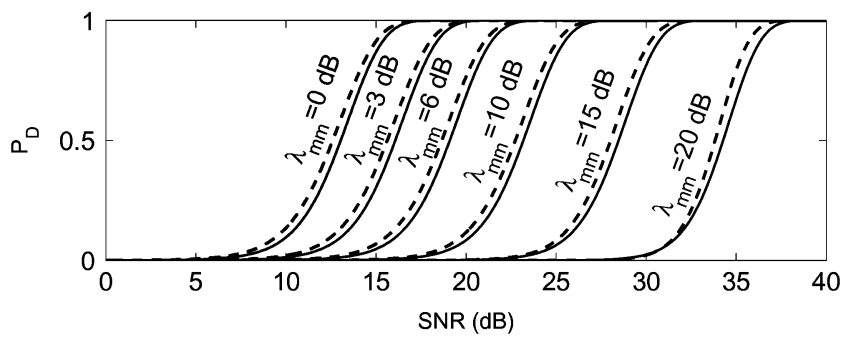

Fig. 11. Performance of the GLRT when $P_{\mathrm{FA}}=10^{-5}$ and mismatch is introduced. The solid curves were calculated using [38] to numerically solve for the doubly-noncentral $\mathrm{F}$ distribution in (17), and the dotted curves were calculated using the normal approximation of (20). Each set of curves (solid and dashed) are labeled with the value of mismatch loss $\lambda_{m m}$ that was assumed.

$\chi_{1}^{2}\left(\lambda_{\ell_{0}} \cos ^{2} \phi\right)$ for large $N M$. For our UWB radar setup, typical values of $M$ are on the order of 10 and typical values of $N$ are on the order of 100, so $N M$ is on the order of 1000 . Since $N M$ is large, we use the $\chi^{2}$ approximation in the following analysis. If we take the square root of the $\chi^{2}$ approximation to the test statistic distribution, then we can analyze the approximate performance of the matched and mismatched GLRT with a convenient Gaussian distribution

$$
\sqrt{\left(1+\frac{\lambda_{\ell_{0}} \sin ^{2} \phi}{N M-1}\right) T_{\ell}} \sim \mathcal{N}\left(\sqrt{\lambda_{\ell_{0}} \cos ^{2} \phi}, 1\right) .
$$

Hence, the probability of detection is approximated by

$$
P_{D}\left(\lambda_{\ell_{0}} \cos ^{2} \phi\right)=\int_{\gamma^{\prime}}^{\infty} \mathrm{d} \mathcal{N}\left(\sqrt{\lambda_{\ell_{0}} \cos ^{2} \phi}, 1\right)
$$

where $\gamma^{\prime}$ is selected to satisfy a given probability of false alarm, $P_{\mathrm{FA}}$. The advantage of (20) over (17) lies in the transparent relationship between the probability of correct detection and mismatch loss. With the Gaussian approximation, mismatch loss simply shifts the mean of the Gaussian distribution to the left and the reduction in $P_{D}$ is straightforward to calculate. In contrast, using the doubly noncentral $\mathrm{F}$ distribution, mismatch loss effects both the numerator and denominator of the doubly noncentral $\mathrm{F}$ variable, and calculating the reduction in $P_{D}$ due to mismatch loss requires costly computational methods or numerical approximations such as the one described in [38].

Fig. 11 shows a plot of $P_{D}$ as a function of $\operatorname{SNR}\left(\lambda_{\ell_{0}}\right)$ and mismatch loss $\left(\lambda_{m m}\right)$ for $P_{\mathrm{FA}}=10^{-5}, \nu_{1}=1$, and $\nu_{2}=305$. The solid curves are calculated using (17) where the doubly noncentral $\mathrm{F}$ distribution is approximated according to [38] and the dotted curves are calculated using the Gaussian approximation for $P_{D}$ given by (20). The solid and dotted curves are in good agreement for both the matched and mismatched cases. In the matched case $\left(\lambda_{m m}=0 \mathrm{~dB}\right), P_{D}$ is greater than 0.95 when SNR is greater than $16 \mathrm{~dB}$. As mismatch is introduced, $\lambda_{m m}$ increases from zero and the resulting $P_{D}$ curves are shown for $\lambda_{m m}=3,6,10,15$, and $20 \mathrm{~dB}$. Introducing mismatch into the GLRT approximately translates the matched $P_{D}$ curve to the right by $\lambda_{m m}$ and simply scales the mean of the Gaussian distribution.

Next we present several examples that illustrate the effects of some common sources of physical mismatch in the GLRT. For these examples, we use the 2-D signal templates described in

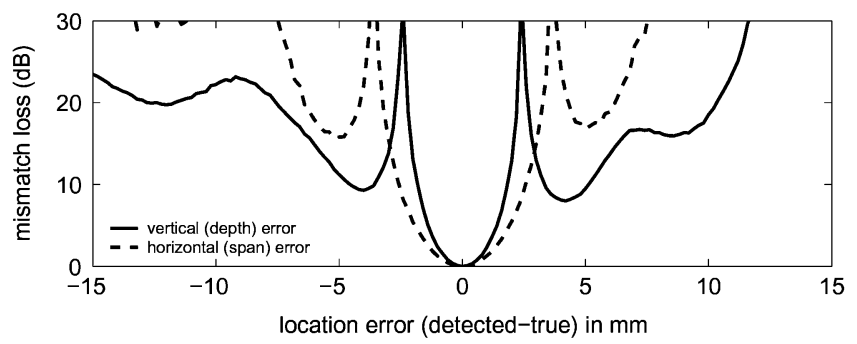

Fig. 12. Mismatch loss as a function of location error for a 2-mm diameter test scatterer $(5.0 \mathrm{~cm}, 2.1 \mathrm{~cm})$. The location error is the horizontal or vertical offset between the true scatterer location and the test location.

Section IV.A, whitened by a sample clutter covariance matrix that is approximately matched to the assumed dielectric properties of the background medium. Recall that the signal templates are parameterized in Section IV.A by the scatterer location, scatterer diameter, and the dielectric properties of the scatterer and background medium. We give examples of the mismatch loss incurred for location errors alone and for compound errors where both location and one other scattering parameter are incorrectly assumed in the GLRT.

The first type of mismatch that we consider is an error in the tumor location. Consider a scenario where coarse spatial sampling is used to generate the image of detected scatterers. If the sampling grid is too coarse, then a small tumor whose true location falls between grid points might not be detected due to a significant mismatch loss. We justify our $1 \mathrm{~mm}$ sampling grid in this example by letting the true scatterer parameters correspond to a 2-mm-diameter tumor centered under the conformal antenna array at a depth of $2.1 \mathrm{~cm}$. The permittivity and conductivity of normal and malignant tissue correspond to the average dielectric properties of a relatively dense phantom, $n 6$ in Table I. These are the same conditions used for the image in Fig. 3(b). The parameters assumed by the GLRT are matched to the true values except for the scatterer location which is varied in either the depth or span direction. In Fig. 12 a plot of mismatch loss as a function of scatterer location error is shown when all other scattering parameters are perfectly matched. The two curves in the plot represent location errors in the depth (solid line) and span (dotted line) directions. As expected, the mismatch loss is zero when no location error exists and the loss increases over a main lobe as the location error deviates from zero. For a 1-mm sampling grid, the largest location error of $0.5 \mathrm{~mm}$, which occurs half-way between adjacent sample points, introduces less than $0.9 \mathrm{~dB}$ loss for depth errors and less than $0.3 \mathrm{~dB}$ loss for span errors. These small losses suggest that even when a tumor falls half-way between grid sampling points, it is almost as likely to be detected as when a tumor falls exactly on a grid sampling point. The curve in Fig. 12 is also useful for distinguishing main and sidelobe peaks in an image of detected scatterers. The local minima that are apparent in the mismatch loss for location errors $\pm 4 \mathrm{~mm}$ in the depth direction indicate significant correlation between the templates at these locations and the templates at the true location, so we expect to see secondary peaks at these locations in the image of detected scatterers. As predicted, the small sidelobes seen in Fig. 3(b) that occur below and to the right of the mainlobe of the detected scatterer are approximately $4 \mathrm{~mm}$ from the peak pixel. The absence of left and top sidelobes is not 


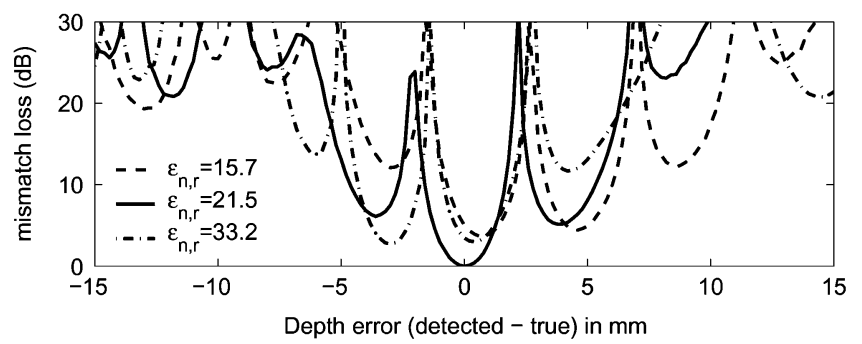

Fig. 13. Mismatch loss as a function of both scatterer location and dielectric properties of the background medium. The 2-mm diameter test scatterer is centered at $(5.0 \mathrm{~cm}, 2.1 \mathrm{~cm})$ in a background medium with $\epsilon_{r, n}=21.50$ while the true scatterer density and location are varied. Location errors are restricted to offsets along the depth axis.

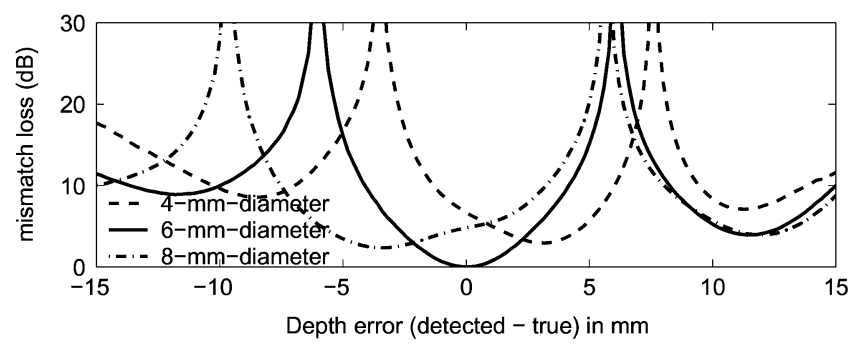

Fig. 14. Mismatch loss as a function of both scatterer location and size. The test scatterer has $6-\mathrm{mm}$ diameter centered at $(5.0 \mathrm{~cm}, 2.1 \mathrm{~cm})$ while the true scatterer diameter and location are varied. Location errors are restricted to offsets along the depth axis.

inconsistent with the curves in Fig. 12. In fact those sidelobes do exist, but have slightly lower test statistic values that fall below the threshold and thus are not detected. Similarly for Figs. 4, 5, and 6 , the sidelobe locations can be predicted by producing corresponding mismatch loss curves for these cases.

Next we consider the case where the GLRT assumes incorrect values for the average dielectric properties of normal breast tissue. We let the true scattering scenario consist of a 2-mmdiameter tumor at a depth of $2.1 \mathrm{~cm}$ centered under the antenna array, and the background medium has dielectric constant $\epsilon_{r, n}=21.50$ and conductivity $\sigma=1.66 \mathrm{~S} / \mathrm{m}$ at $6 \mathrm{GHz}$. The assumed scattering scenario is matched to the true scattering scenario except for the scatterer depth and the dielectric properties of the background medium which we allow to vary. Fig. 13 depicts the mismatch loss as a function of location error for each of three assumed dielectric constants: the dashed line represents an underestimated density GLRT, $\epsilon_{r, n}=15.65$ and $\sigma=1.03$ at $6 \mathrm{GHz}$; the solid line represents the matched density GLRT, $\epsilon_{r, n}=21.50$ and $\sigma=1.66$ at $6 \mathrm{GHz}$; and the dash-dot line represents the overestimated density GLRT, $\epsilon_{n}=27.35$ and $\sigma=2.29$ at $6 \mathrm{GHz}$. This plot suggests that when the density and attenuation of normal breast tissue is underestimated, a scatterer is still likely to be detected since the mismatch loss has two local minima at only a few $\mathrm{dB}$ loss, but the location of the scatterer will appear deeper than the true depth of the scatterer. Conversely, if we overestimate the density of normal breast tissue, the location of the scatterer will most likely appear shallower than the true depth of the scatterer, but again the mismatch loss is small and the scatterer is still likely to be detected.

The last type of mismatch that we consider in this section is the combination of scatterer size and depth errors. Fig. 14 depicts the mismatch loss when both the diameter and depth of the scatterer are mismatched. The plot illustrates that mismatch in scatterer size introduces a localization error in the depth axis. That is, the peak of the test statistic occurs at the incorrect depth, but as before the scatterer is still likely to be detected since the mismatch loss is low at a slightly offset location.

\section{SUMMARY}

We have explored the feasibility of the GLRT for UWB microwave detection of strong scatterers in the breast such as malignant tumors using simulated backscatter from 2-D MRI-derived breast phantoms and a 3-D homogeneous numerical breast phantom, and experimental backscatter from multilayer physical breast phantoms. We address the problem of clutter, which occurs in the backscatter data due to normal heterogeneity of the breast, with a statistical model to account for the variability in the structure of normal breast tissue from patient to patient. The clutter covariance matrix is estimated by a sample covariance matrix obtained from clutter observations of numerous realistic numerical breast phantoms. We show that the GLRT together with the proposed Gaussian clutter model is adept at detecting and localizing small tumors in numerical breast phantoms. Several challenging numerical breast phantoms with reduced malignant-to-normal dielectric contrast are also considered and the GLRT successfully detects and localizes 2-mm-diameter tumors in the realistic numerical breast phantoms with dielectric contrasts as low as 1.43:1. Further development of statistical data models may lead to improvements in detection performance for the two heterogeneously dense cases considered in this paper where the tumor was not correctly detected. Our experimental breast phantoms are homogeneous, so the backscatter data is clutter-free and modeled by a deterministic signal plus white Gaussian noise. Again the GLRT is able to accurately detect and localize multiple small tumors in the phantom, even for reduced dielectric contrast. For the final phantom that we consider, a 3-D numerical breast phantom with homogeneous normal tissue, the small tumor is again correctly detected and well localized in the image. We conclude with an analysis of the performance of the GLRT when mismatch is introduced and characterize the loss in performance by the cosine of the geometric angle between the actual and assumed signal vectors. For data with high SNR, moderate to severe levels of mismatch are well tolerated by the GLRT. Often the result of mismatch is simply a shift in the location of the detected tumor and a few $\mathrm{dB}$ reduction in the test statistic value.

We conclude that the Gaussian clutter-based GLRT is promising as a tool for UWB microwave breast cancer detection. This method of detection warrants studies using more realistic 3-D breast phantoms and further development on the subject of characterization of the scattering properties using the template parameterization.

\section{ACKNOWLEDGMENT}

The authors would like to thank Dr. X. Li for her guidance with the development of the numerical breast phantom and acquisition of backscatter data. They also thank E. Bond for providing the 2-D signal templates. 


\section{REFERENCES}

[1] Mammography and Beyond: Developing Techniques for the Early Detection of Breast Cancer. Washington, DC: National Academy, 2000.

[2] P. T. Huynh, A. M. Jarolimek, and S. Daye, "The false-negative mammogram," Radiography, vol. 18, no. 5, pp. 1137-1154, 1998.

[3] S. S. Chaudhary, R. K. Mishra, A. Swarup, and J. M. Thomas, "Dielectric properties of normal and malignant human breast tissues at radiowave and microwave frequencies," Ind. J. Biochem. Biophys., vol. 21, pp. 76-79, Feb. 1984.

[4] A. J. Surowiec, S. S. Stuchly, J. R. Barr, and A. Swarup, "Dielectric properties of breast carcinoma and the surrounding tissues," IEEE Trans. Biomed. Eng., vol. 35, pp. 257-263, Apr. 1988.

[5] W. T. Joines, Y. Z. Dhenxing, and R. L. Jirtle, "The measured electrical properties of normal and malignant human tissues from 50 to $900 \mathrm{MHz}$," Med. Phys., vol. 21, pp. 547-550, Apr. 1994

[6] L. V. Wang, X. Zho, H. Sun, and G. Ku, "Microwave-induced acoustic imaging of biological tissues," Rev. Sci. Instrum., vol. 70, pp. 3744-3748, 1999

[7] R. A. Kruger, K. K. Kopecky, A. M Aisen, D. R. Reinecke, G. A. Kruger, and W. L. Kiser Jr., "Thermoacoustic CT with radio waves: A medical imaging paradigm," Radiol., vol. 211, pp. 275-278, 1999.

[8] R. A. Kruger, K. D. Miller, H. E. Reynolds, W. L. Kiser Jr., D. R. Reinecke, and G. A. Kruger, "Breast cancer in vivo: Contrast enhancement with thermoacoustic CT at $434 \mathrm{MHz}$-Feasibility study," Radiol., vol. 211, pp. 279-283, 2000.

[9] P. M. Meaney and K. D. Paulsen, "Nonactive antenna compensation for fixed-array microwave imaging: Part II-Imaging results," IEEE Trans. Med. Imag., vol. 18, no. 6, pp. 508-518, Jun. 1999.

[10] P. M. Meaney, M. W. Fanning, D. Li, S. P. Poplack, and K. D. Paulsen, "A clinical prototype for active microwave imaging of the breast," IEEE Trans. Microwave Theory Tech., vol. 48, no. 11, pp. 1841-1853, Nov. 2000.

[11] D. Li, P. M. Meaney, T. Raynolds, S. A. Pendergrass, M. W. Fanning, and K. D. Paulsen, "Parallel-detection microwave spectroscopy system for breast imaging," Rev. Sci. Instrum., vol. 75, pp. 2305-2313, 2004.

[12] S. P. Poplack, P. M. Meaney, A. Hartov, K. D. Paulsen, T. D. Torsteson, M. R. Grove, S. K. Soho, and W. A. Wells, "Electromagnetic breast imaging: Average tissue property values in women with negative clinical findings," Radiol., vol. 231, no. 2, pp. 571-580, May 2004.

[13] A. E. Souvorov, A. E. Bulyshev, S. Y. Semenov, R. H. Svenson, and G. P. Tatsis, "Two-dimensional computer analysis of a microwave flat antenna array for breast cancer tomography," IEEE Trans. Microwave Theory Tech., vol. 48, no. 8, pp. 1413-1415, Aug. 2000.

[14] A. E. Bulyshev, S. Y. Semenov, A. E. Souvorov, R. H. Svenson, A. G. Nazarov, Y. E. Sizov, and G. P. Tatsis, "Computational modeling of three-dimensional microwave tomography of breast cancer," IEEE Trans. Biomed. Eng., vol. 48, no. 9, pp. 1053-1056, Sep. 2001.

[15] Q. H. Liu, Z. Q. Zhang, T. T. Wang, J. A. Bryan, G. A. Ybarra, L. W. Nolte, and W. T. Joines, "Active microwave imaging I-2-D forward and inverse scattering methods," IEEE Trans. Microwave Theory Tech., vol. 50, no. 1, pp. 123-133, Jan. 2002

[16] Z. Q. Zhang, Q. Liu, C. Xiao, E. Ward, G. Ybarra, and W. T. Joines, "Microwave breast imaging: 3-D forward scattering simulation," IEEE Trans. Biomed. Eng., vol. 50, no. 10, pp. 1180-1189, Oct. 2003.

[17] S. C. Hagness, A. Taflove, and J. E. Bridges, "Two-dimensional FDTD analysis of a pulsed microwave confocal system for breast cancer detection: Fixed-focus and antenna-array sensors," IEEE Trans. Biomed. Eng., vol. 45, no. 12, pp. 1470-1479, Dec. 1998.

[18] — - "Three-dimensional FDTD analysis of a pulsed microwave confocal system for breast cancer detection: Design of an antenna-array element," IEEE Trans. Antennas Propagat., vol. 47, no. 5, pp. 783-791, May 1999.

[19] E. C. Fear and M. A. Stuchly, "Microwave detection of breast cancer," IEEE Trans. Microwave Theory Tech., vol. 48, no. 11, pp. 1854-1863, Nov. 2000.

[20] X. Li and S. C. Hagness, "A confocal microwave imaging algorithm for breast cancer detection," IEEE Microwave Wireless Comp. Lett., vol. 11, no. 3, pp. 130-132, Mar. 2001

[21] E. C. Fear, X. Li, S. C. Hagness, and M. Stuchly, "Confocal microwave imaging for breast cancer detection: Localization of tumors in three dimensions," IEEE Trans. Biomed. Eng., vol. 49, no. 8, pp. 812-822, Aug. 2002

[22] E. C. Fear, J. Sill, and M. A. Stuchly, "Experimental feasibility study of confocal microwave imaging for breast tumor detection," IEEE Trans. Microwave Theory Tech., vol. 51, no. 3, pp. 887-892, Mar. 2003.

[23] R. Nilavalan, A. Gbedemah, I. J. Craddock, X. Li, and S. C. Hagness, "Numerical investigation of breast tumor detection using multistatic radar," Electron. Lett., vol. 39, no. 25, pp. 1787-1788, 2003.
[24] M. A. Hernandez-Lopez, M. Quintillan-Gonzalez, S. G. Garcia, A. R Bretones, and R. G. Martin, "A rotating array of antennas for confocal microwave breast imaging," Microw. Opt. Technol. Lett., vol. 39, no. 4, pp. 307-311, 2003.

[25] E. J. Bond, X. Li, S. C. Hagness, and B. D. Van Veen, "Microwave imaging via space-time beamforming for early detection of breas cancer," IEEE Trans. Antennas Propagat., vol. 51, no. 8, pp. 1690-1705, Aug. 2003.

[26] S. K. Davis, E. J. Bond, X. Li, S. C. Hagness, and B. D. Van Veen, "Microwave imaging via space-time beamforming for early-stage breast cancer detection: Beamformer design in the frequency domain," J. Electromagn. Waves Applicat., vol. 17, no. 2, pp. 357-381, 2003.

[27] X. Li, S. K. Davis, S. C. Hagness, D. W. van der Weide, and B. D. Van Veen, "Microwave imaging via space-time beamforming: Experimental investigation of tumor detection in multilayer breast phantoms," IEEE Trans. Microwave Theory Tech., vol. 52, no. 8, pp. 1856-1865, Aug. 2004.

[28] S. M. Kay, Fundamentals of Statistical Signal Processing: Detection Theory. Upper Saddle River, NJ: Prentice-Hall, 1998.

[29] H. Brunzell, "Clutter reduction and object detection in surface penetrating radar," in Proc. Radar 97, Edinburgh, U.K., Oct. 14-16, 1997.

[30] F. Y. Nan and R. D. Nowak, "Generalized likelihood radio detection for fMRI using complex data," IEEE Trans. Med. Imag., vol. 18, no. 4, pp. 320-329, Apr. 1999.

[31] G. R. Grimmett and D. R. Stirzaker, Probability and Random Processes. New York: Oxford Univ. Press, 1992

[32] Y. Benjamini and Y. Hochberg, "Controlling the false discovery rate: A practical and powerful approach to multiple testing," J. R. Statist. Soc. $B$, vol. 57, pp. 289-300, 1995

[33] A. F. Saftlas, R. N. Hoover, L. A. Brinton, M. Szklo, D. R. Olson, M Salane, and J. N. Wolfe, "Mammographic densities and risk of breast cancer," Cancer, vol. 67, pp. 2833-2838, Jun. 1991.

[34] A. Taflove and S. Hagness, Computational Electrodynamics: The FiniteDifference Time-Domain Method, 2nd ed. Boston, MA: Artech House, 2000.

[35] X. Li, S. C. Hagness, M. K. Choi, and D. W. van der Weide, "Numerical and experimental investigation of an ultrawideband ridged pyramidal horn antenna with curved launching plane for pulse radiation," IEEE Antennas Wireless Propagat. Lett., vol. 2, pp. 259-262, 2003.

[36] A. Ishimaru, Electromagnetic Wave Propagation, Radiation, and Scattering. Englewood Cliffs, NJ: Prentice-Hall, 1991

[37] N. L. Johnson, S. Kotz, and N. Balakrishnan, Continuous Univariate Distributions, 2nd ed. New York: Wiley, 1995, vol. 2

[38] R. Butler and M. Paolella, "Calculating the density and distribution function for the singly and doubly noncentral," Stat. Comput., vol. 12, no. 1 pp. 9-16, Jan. 2002.

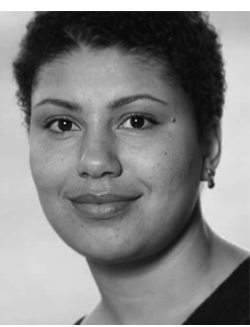

Shakti K. Davis (S'99) received the B.S. degree from New Mexico State University, Las Cruces, in 1999 and the M.S. degree from the University of Wisconsin, Madison, in 2002, both in electrical engineering. She is currently pursuing the Ph.D degree in electrical engineering at the University of Wisconsin, Madison.

She is the recipient of a Predoctoral Fellowship Award for Minority Students from the National Institutes of Health and a current participant in the AT\&T Labs Fellowship Program and the Graduate Engineering Research Scholars program. Her research interests include biomedical applications of signal processing and array signal processing.

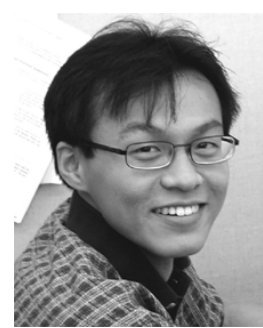

Henri Tandradinata was born in Bali, Indonesia, on May 14, 1979. He received the B.S. degree in May 2002 and the M.S. degree in August 2004 both in electrical engineering from the University of Wisconsin, Madison.

$\mathrm{He}$ is currently a Business Information Specialist with ZS Associates in Evanston, IL. 


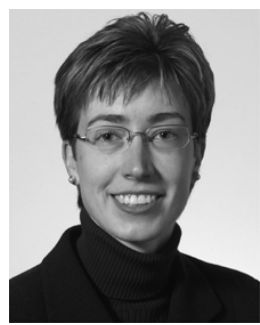

Susan C. Hagness (S'91-M'98-SM'04) received the B.S. degree with highest honors and the Ph.D. degree in electrical engineering from Northwestern University, Evanston, IL, in 1993 and 1998, respectively. While working toward the Ph.D. degree, she was a National Science Foundation (NSF) Graduate Fellow and a Tau Beta Pi Spencer Fellow.

Since August 1998, she has been with the University of Wisconsin, Madison, where she is currently an Associate Professor in the Department of Electrical and Computer Engineering and a Faculty Affiliate of the Department of Biomedical Engineering. Her research interests include FDTD theory and applications in photonics and bioelectromagnetics as well as microwave imaging, sensing, and thermal therapy techniques in biological and medical applications.

Dr. Hagness is an Elected Member of the IEEE Antennas and Propagation Society (AP-S) Administrative Committee and member-at-large of the United States National Committee (USNC) of the International Union of Radio Science (URSI). She is currently an associate editor for the IEEE ANTENNAS AND WiReless Propagation LetTers. She was the recipient of the Presidential Early Career Award for Scientists and Engineers presented by the White House in 2000. In 2002, she received the Booker Fellowship Award from the USNC/URSI. She was also named one of the 100 top young innovators in science and engineering in the world by the Massachusetts Institute of Technology (MIT) Technology Review magazine. In 2003, she received the University of Wisconsin Emil Steiger Distinguished Teaching Award. She received the IEEE Engineering in Medicine and Biology Society Early Career Achievement Award in 2004 and the International Union of Radio Science (URSI) Isaac Koga Gold Medal in 2005.

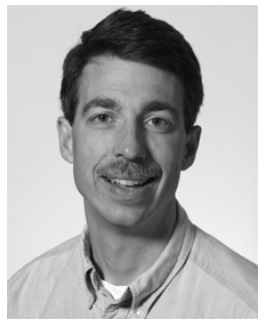

Barry D. Van Veen (S'81-M'86-SM'97-F'02) was born in Green Bay, WI. He received the B.S. degree from Michigan Technological University, Houghton, in 1983 and the Ph.D. degree from the University of Colorado, Boulder, in 1986, both in electrical engineering. He was an ONR Fellow while working on the Ph.D. degree.

In the spring of 1987, he was with the Department of Electrical and Computer Engineering, University of Colorado, Boulder. Since August of 1987, he has been with the Department of Electrical and Computer Engineering, University of Wisconsin, Madison, and currently holds the rank of Professor. His research interests include signal processing for sensor arrays, wireless communications, and biomedical applications of signal processing. He coauthored Signals and Systems (New York: Wiley, 1999) with S. Haykin.

Dr. Van Veen was a recipient of a 1989 Presidential Young Investigator Award from the National Science Foundation and a 1990 IEEE Signal Processing Society Paper Award. He served as an Associate Editor for the IEEE Transactions on Signal Processing and on the IEEE Signal Processing Society's Statistical Signal and Array Processing Technical Committee and the Sensor Array and Multichannel Technical Committee. He received the Holdridge Teaching Excellence Award from the ECE Department, University of Wisconsin, Madison, in 1997. 